Geometry \& Topology

Volume 9 (2005) 121-162

Published: 28 December 2004

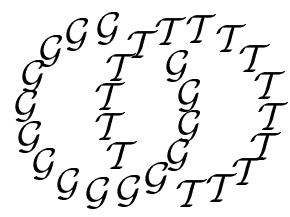

\title{
Homotopy properties of Hamiltonian group actions
}

\author{
JAREK KȨDRA \\ Dusa McDufF
}

Institute of Mathematics US, Wielkopolska 15, 70-451 Szczecin, Poland and Mathematical Institute Polish Academy of Sciences

Śniadeckich 8, 00-950 Warszawa, Poland

and

Department of Mathematics, Stony Brook University

Stony Brook, NY 11794-3651, USA

Email: kedra@univ.szczecin.pl and dusa@math.sunysb.edu

http://www.univ.szczecin.pl/ kedra, http://www.math.sunysb.edu/ dusa

\begin{abstract}
Consider a Hamiltonian action of a compact Lie group $G$ on a compact symplectic manifold $(M, \omega)$ and let $\mathcal{G}$ be a subgroup of the diffeomorphism group Diff $M$. We develop techniques to decide when the maps on rational homotopy and rational homology induced by the classifying map $B G \rightarrow B \mathcal{G}$ are injective. For example, we extend Reznikov's result for complex projective space $\mathbb{C P}^{n}$ to show that both in this case and the case of generalized flag manifolds the natural map $H_{*}(B S U(n+1)) \rightarrow H_{*}(B \mathcal{G})$ is injective, where $\mathcal{G}$ denotes the group of all diffeomorphisms that act trivially on cohomology. We also show that if $\lambda$ is a Hamiltonian circle action that contracts in $\mathcal{G}:=\operatorname{Ham}(M, \omega)$ then there is an associated nonzero element in $\pi_{3}(\mathcal{G})$ that deloops to a nonzero element of $H_{4}(B \mathcal{G})$. This result (as well as many others) extends to c-symplectic manifolds $(M, a)$, ie, $2 n$-manifolds with a class $a \in H^{2}(M)$ such that $a^{n} \neq 0$. The proofs are based on calculations of certain characteristic classes and elementary homotopy theory.
\end{abstract}

AMS Classification numbers Primary: 53C15

Secondary: 53D05, 55R40, 57R 17

Keywords: Symplectomorphism, Hamiltonian action, symplectic characteristic class, fiber integration

Proposed: Ralph Cohen

Received: 30 April 2004

Seconded: Leonid Polterovich, Frances Kirwan

Revised: 22 December 2004

(C) Geometry ${ }^{6}$ Topology Publications 


\section{Introduction}

\subsection{Overview of results}

This paper studies the homotopy type of the $\operatorname{group} \operatorname{Symp}(M, \omega)$ of symplectomorphisms of a closed symplectic manifold $(M, \omega)$ onto itself. It was noticed from the beginning of the modern development of symplectic topology that many basic results can be expressed in terms of properties of this group. For instance, one way to express symplectic rigidity is to observe that the group $\operatorname{Symp}(M, \omega)$ is closed in the $C^{0}$ (or uniform) topology on the diffeomorphism group $\operatorname{Diff}(M)$, rather than just in the $C^{1}$-topology as one would expect.

In dimension two, it follows from Moser's theorem that the group of oriented area preserving (or symplectic) diffeomorphisms of any surface $(\Sigma, \omega)$ is homotopy equivalent to the full group of orientation preserving diffeomorphisms $\operatorname{Diff}^{+}(\Sigma)$. Moreover, its homotopy type is well known. For example, in the case of the 2 -sphere, Smale showed that $\operatorname{Symp}\left(S^{2}, \omega\right) \simeq \operatorname{Diff}^{+}\left(S^{2}\right)$ is homotopy equivalent to the group $S O(3)$ of rotations.

In dimension four, the two groups $\operatorname{Symp}(M, \omega)$ and $\operatorname{Diff}^{+}(M)$ are very different. Very little is known about the homotopy groups of $\operatorname{Diff}^{+}(M)$ (even for the $4-$ sphere) while in certain cases it has turned out that $\operatorname{Symp}(M, \omega)$ is accessible. Using the method of pseudoholomorphic curves, Gromov 10 proved that the identity component of the group $\operatorname{Symp}\left(S^{2} \times S^{2}, \omega_{1}\right)$ is homotopy equivalent to to $S O(3) \times S O(3)$. Here $\omega_{\lambda}$ denotes the product symplectic form in which the first sphere $S^{1} \times\{p\}$ has area equal to $\lambda$ and the second $\{p\} \times S^{2}$ has area equal to 1 . Hence in the above situation both spheres have equal size. Similarly, the symplectomorphism group of $\mathbb{C P}^{2}$ with its standard symplectic form deformation retracts to the group of linear isometries $P S U(3)$.

Abreu, Anjos and McDuff [1, 4] extended Gromov's work, studying the family of groups $\operatorname{Symp}\left(S^{2} \times S^{2}, \omega_{\lambda}\right)$ for $\lambda>1$. They found that although these groups are not homotopy equivalent to any compact Lie group, their rational homotopy is detected by actions of compact Lie groups. More precisely, the rational homotopy of $\operatorname{Symp}\left(S^{2} \times S^{2}, \omega_{\lambda}\right)$ is nontrivial in degrees 1,3 and $4 k$ where $k$ is the largest integer $<\lambda$. The generator in degree one is represented by a circle action, the two generators in degree three are represented by actions of $S O(3)$ and the last generator is a certain higher order Samelson product of the previous ones 1 This set of results culminated in a theorem of Anjos-Granja

\footnotetext{
${ }^{1}$ Recall that if $G$ is a topological group then the Samelson product $\langle\rangle:, \pi_{k}(G) \times$ $\pi_{m}(G) \rightarrow \pi_{k+m}(G)$ is defined by$$
\langle\alpha, \beta\rangle: S^{k+m}=S^{k} \times S^{m} / S^{k} \vee S^{m} \rightarrow G . \quad[s, t] \mapsto \alpha(s) \beta(t) \alpha(s)^{-1} \beta(t)^{-1} .
$$ 
[5] stating that when $1<\lambda \leq 2$ the group $\operatorname{Symp}\left(S^{2} \times S^{2}, \omega_{\lambda}\right)$ is homotopy equivalent to the topological amalgamated product

$$
(S O(3) \times S O(3)) *_{S O(3)}\left(S O(3) \times S^{1}\right),
$$

where $S O(3)$ maps to the diagonal subgroup of $S O(3) \times S O(3)$ and is included as the first factor in $S O(3) \times S^{1}$.

In all these cases the rational homotopy of the symplectomorphism group is determined by some compact subgroups arising from Lie group actions that preserve a Kähler metric on the underlying manifold. As a first step towards understanding what happens in higher dimensions, we look at manifolds that admit an effective symplectic action by a compact connected Lie group $G$ and see how much of the (rational) homotopy of $G$ remains visible in $\operatorname{Symp}(M, \omega)$, either directly in the sense that the induced map on $\pi_{*} \otimes \mathbb{Q}$ is injective, or indirectly in the sense that there is some associated nontrivial element in $\pi_{*}(\operatorname{Symp}(M, \omega))$ (such as a secondary Samelson product). Reznikov [22] proved some initial results in this direction. By defining and calculating some new characteristic classes, he showed that any nontrivial homomorphism $S U(2) \rightarrow \operatorname{Symp}(M, \omega)$ induces an injection on $\pi_{3}$. He also showed that the canonical map of $S U(n+1)$ into $\operatorname{Symp}\left(\mathbb{C P}^{n}\right)$ induces an injection on rational homotopy. In fact, he proved the sharper result that for $i>1$ the Chern classes $c_{i} \in H^{2 i}(B S U(n+1) ; \mathbb{R})$ have natural extensions to classes in $H^{2 i}\left(B \operatorname{Symp}\left(\mathbb{C P}^{n}\right) ; \mathbb{R}\right)$. Thus the induced map

$$
H_{*}(B S U(n+1) ; \mathbb{Q}) \rightarrow H_{*}\left(B \operatorname{Symp}\left(\mathbb{C P}^{n}\right) ; \mathbb{Q}\right)
$$

is injective 2 Similar results were obtained for certain toric manifolds by Januszkiewicz-Kẹdra in [13].

In Section 3 of this paper we show that Reznikov's characteristic classes are closely related to those used by Januszkiewicz-Kędra and to the $\kappa$-classes of Miller-Morita-Mumford. In particular they may be defined without using any geometry, and so they extend to cohomologically symplectic (or c-symplectic) manifolds $(M, a)$, ie, to pairs consisting of a closed oriented $2 n$-manifold $M$ together with a cohomology class $a \in H^{2}(M ; \mathbb{R})$ such that $a^{n}>0$. However, if

\footnotetext{
${ }^{2}$ The injectivity of $r_{*}: \pi_{*}(G) \otimes \mathbb{Q} \rightarrow \pi_{*}(\mathcal{G}) \otimes \mathbb{Q}$ is equivalent to that of the induced map on classifying spaces $R_{*}: \pi_{*}(B G) \otimes \mathbb{Q} \rightarrow \pi_{*}(B \mathcal{G}) \otimes \mathbb{Q}$. However this does not mean that the induced map on rational homology $H_{*}(B G ; \mathbb{Q}) \rightarrow H_{*}(B \mathcal{G} ; \mathbb{Q})$ must be injective, because the rational Hurewicz map $h: \pi_{*}(B \mathcal{G}) \otimes \mathbb{Q} \rightarrow H_{*}(B \mathcal{G}) \otimes \mathbb{Q}$ is not always injective when $\mathcal{G}=\operatorname{Symp}(M, \omega)$, though it is for connected Lie groups. Equivalently, the rational Whitehead product need not vanish on $B \mathcal{G}$ : see Section [2]
} 
$H^{1}(M ; \mathbb{R}) \neq 0$, these classes do not live on the analog of the full symplectomorphism group but rather on the analog of its Hamiltonian $3 \operatorname{subgroup} \operatorname{Ham}(M, \omega)$. If $H^{1}(M ; \mathbb{R})=0$ they can be extended significantly further, see Remark 1.2 and Section 3 .

We extend Reznikov's work in two directions, first by looking at homogeneous spaces other than $\mathbb{C P}^{n}$, and second by considering homotopy groups in dimensions other than three. The first is fairly straightforward; for example we prove in Proposition 4.8 that the action of $S U(n)$ on generalized flag manifolds induces an injection of rational homology at the classifying space level. However, the second is more delicate because it is not true that every inclusion $U(k) \rightarrow \operatorname{Symp}(M, \omega)$ induces an injection on $\pi_{i} \otimes \mathbb{Q}$ for $i \neq 3$. As shown in more detail in Section 4.1 our counterexamples are based on the existence of homomorphisms $U(m) \rightarrow U(n)$ that kill homotopy. For instance the homomorphism

$$
U(m) \rightarrow U(m) \times U(m) \subset U(2 m), \quad A \mapsto(A, \bar{A})
$$

(where $\bar{A}$ is complex conjugation) kills homotopy in dimension $4 k+1$. One cannot remedy this by considering maximal compact $\operatorname{subgroups}$ of $\operatorname{Symp}(M, \omega)$ : McDuff-Tolman [20] construct a toric Kähler structure on the product $\mathbb{C P}^{1} \times$ $\mathbb{C P}^{2}$ whose isometry group $G$ is maximal in $\operatorname{Symp}(M, \omega)$ but is such that $\pi_{1}(G) \otimes \mathbb{Q}$ does not inject. Therefore the homotopy of $G$ need not be directly visible in Symp. However, we shall see in Theorem 1.1 that in the case of a null homotopic circle action one can use the null homotopy itself to define an element $\rho \in \pi_{3}(\operatorname{Symp}(M, \omega))$ that never vanishes. This is the simplest case of a general construction that is developed in Section 2, In Section 5 we discuss further properties of this element $\rho$, looking in particular at its image in $\pi_{3}(M)$ under the pointwise evaluation map $\operatorname{Symp}(M, \omega) \rightarrow M$.

This paper uses elementary methods from algebraic topology. For recent results on the homotopy of $\operatorname{Symp}(M, \omega)$ that use deeper, more analytic techniques, readers might consult McDuff 16 or Seidel 23. In the rest of this introduction we state our main results in more detail. We denote by $H_{*}(M), H^{*}(M)$ (co)homology with real or rational (rather than integral) coefficients.

${ }^{3}$ This consists of the time- 1 maps $\phi_{1}^{H}$ of Hamiltonian flows $\phi_{t}^{H}, t \in[0,1]$. These paths are generated by time dependent Hamiltonian functions $H_{t}: M \rightarrow \mathbb{R}$ via the recipe $\omega\left(\dot{\phi}_{t}^{H}, \cdot\right)=d H_{t}(\cdot)$. If $H^{1}(M ; \mathbb{R})=0$, then $\operatorname{Ham}(M, \omega)$ is just the identity component $\operatorname{Symp}_{0}(M, \omega)$ of the symplectomorphism group. In general, it is the kernel of the flux homomorphism (3.2): for further background information and references, see for example McDuff-Salamon [18]. 


\section{$1.2 \quad$ Circle actions}

Let $\mathcal{G}$ be a topological group and $\lambda: S^{1} \rightarrow \mathcal{G}$ a nonconstant homomorphism that represents the zero element in $\pi_{1}(\mathcal{G})$ and so extends to a map $\widetilde{\lambda}: D^{2} \rightarrow \mathcal{G}$. (For short, we say that $S^{1}$ is inessential in $\mathcal{G}$.) Define $\rho \in \pi_{3}(\mathcal{G})$ by setting

$$
\begin{gathered}
\rho: S^{3}=\left(D^{2} \times S^{1}\right) /\left(\left(D^{2} \times\{1\}\right) \vee\left(\partial D^{2} \times S^{1}\right)\right) \rightarrow \mathcal{G}, \\
(z, t) \mapsto\langle\widetilde{\lambda}(z), \lambda(t)\rangle,
\end{gathered}
$$

where the bracket $\langle\phi, \psi\rangle$ represents the commutator $\phi \psi \phi^{-1} \psi^{-1}$. Observe that the map $D^{2} \times S^{1} \rightarrow \mathcal{G}$ descends to $S^{3}$ precisely because it contracts the boundary $\partial D^{2} \times S^{1}$ to a point. Thus it is crucial here that $G=S^{1}$ is abelian and that $\lambda$ is a homomorphism.

Theorem 1.1 Let $(M, \omega)$ be a closed symplectic manifold of dimension $2 n$ and set $\mathcal{G}:=\operatorname{Ham}(M, \omega)$. Let $\lambda: S^{1} \rightarrow \mathcal{G}$ be a nontrivial homomorphism that is inessential in $\mathcal{G}$. Then the element $\rho \in \pi_{3}(\mathcal{G})$ defined above is independent of the choice of extension $\widetilde{\lambda}$ and has infinite order. Moreover, the corresponding element $\bar{\rho} \in \pi_{4}(B \mathcal{G})$ has nonzero image in $H_{4}(B \mathcal{G} ; \mathbb{Q})$.

Remark 1.2 (i) To see that $\rho$ is independent of the choice of $\widetilde{\lambda}$, observe that any two extensions differ by an element $\beta \in \pi_{2}(\mathcal{G})$. This changes $\rho$ by the Samelson product $\langle\beta, \lambda\rangle$, which vanishes in $\mathcal{G}$ since $\lambda=0$ in $\pi_{1}(\mathcal{G})$.

(ii) If $\lambda$ is a smooth inessential circle action on $M$ then one can use formula (1.1) to define an element $\rho \in \pi_{3}(\operatorname{Diff}(M))$. However, in this generality we have no way of proving that $\rho \neq 0$.

(iii) We detect the nontriviality of $\bar{\rho}$ by using the characteristic classes of Reznikov [22] and Januszkiewicz-Kędra [13. These classes extend beyond the Hamiltonian group $\operatorname{Ham}(M, \omega)$ to appropriate topological monoids $\mathcal{H}$ that act on $M$. For example, we show in Corollary 3.7 that if $\lambda$ is a smooth action on a simply connected c-symplectic manifold $(M, a)$ that is inessential in the topological monoid $\mathcal{H}_{a}$ formed by all smooth homotopy equivalences $M \rightarrow M$ that fix the class $a$ then $\bar{\rho}$ has nonzero image in $H_{4}\left(B \mathcal{H}_{a}\right)$.

(iv) Our result extends work by Reznikov in the following way. Reznikov proved in 22] that any Hamiltonian action of $S U(2)$ induces a nonzero map on $\pi_{3} \otimes \mathbb{Q}$. Moreover, Lemma 2.5 below implies that the element $\rho$ created from any circle subgroup of $S U(2)$ lies in the image of $\pi_{3}(S U(2))$. Thus the nontriviality statement in Theorem 1.1 follows from Reznikov's work in the 
case when the circle $\lambda$ contracts in $\operatorname{Ham}(M, \omega)$ by virtue of the fact that it is contained in a simply connected Lie subgroup $G$ of $\operatorname{Ham}(M, \omega)$.

(v) Let $\mathrm{ev}_{*}: \pi_{*}(\mathcal{G}) \rightarrow \pi_{*}(M)$ denote the map obtained by evaluating at the base point $p$. By looking at the $S U(2)$-action on $M:=\mathbb{C P}^{n}$ for $n=1,2$ and using Lemma 2.5 as in (iv) above, one sees that the element $\operatorname{ev}_{*}(\rho) \in \pi_{3}(M)$ is sometimes zero and sometimes nonzero. We show in Proposition 5.3 that if $H^{1}(M)=0$ then $\operatorname{ev}_{*}(\rho) \neq 0$ only if there is a nonzero quadratic relation $\sum_{i j} c_{i} c_{j}=0$ among the classes $c_{i} \in H^{2}(M)$. In the symplectic case this is no surprise since it follows from the work of Lalonde-McDuff [14] that the map

$$
h \circ \mathrm{ev}_{*}: \pi_{*}(\operatorname{Ham}(M, \omega)) \rightarrow H_{*}(M ; \mathbb{Q})
$$

is zero, where $h$ denotes the Hurewicz homomorphism. Hence, by minimal model theory, any element in the image of $\mathrm{ev}_{*}$ in this low degree must give rise to a relation in $H^{*}(M)$. However, the arguments in [14] do not apply in the c-symplectic case.

A Hamiltonian $S^{1}$-action always has fixed points $p$ and one can consider its image in the subgroup Diff $p$ of diffeomorphisms that fix $p$. When $H^{1}(M) \neq 0$ we shall need to consider the corresponding subgroup $\mathcal{G}_{p}$ of $\mathcal{G}:=\operatorname{Ham}(M, \omega)$ which is the fiber of the evaluation map $\mathcal{G} \rightarrow M$ at $p$. The following result is proved in Section 3.2

Lemma 1.3 Suppose that $\lambda$ is a Hamiltonian circle action with moment map $H: M \rightarrow \mathbb{R}$, and set $\mathcal{G}:=\operatorname{Ham}(M, \omega)$. Then $\lambda$ is essential in $\mathcal{G}_{p}$ for every fixed point $p$ such that $\int_{M}(H-H(p)) \omega^{n} \neq 0$. In particular, $\lambda$ is essential in $\mathcal{G}_{p}$ if $H$ assumes its maximum or minimum at $p$.

A similar statement holds in the c-Hamiltonian case.

\subsection{Higher homotopy groups}

The proof of Theorem 1.1 is based on a general construction of a secondary product $\{\cdot \dot{\cdot} \cdot\}$ on the homotopy of topological groups. Given a point $p \in M$ we shall write $G_{p}, \mathcal{G}_{p}$ for the subgroups of $G, \mathcal{G}$ that fix $p$.

Let $r: G \rightarrow \mathcal{G}$ be a continuous homomorphism. Consider the induced map

$$
r_{k-1}: \pi_{k-1}\left(G_{p}\right) \otimes \mathbb{Q} \rightarrow \pi_{k-1}(\mathcal{G}) \otimes \mathbb{Q}
$$

\footnotetext{
${ }^{4}$ Our conventions are that $G$ denotes a compact Lie group while $\mathcal{G}$ (usually) denotes an infinite dimensional group such as Symp or Diff.
} 
In Section 2 we construct an element

$$
\left\{f, f^{\prime}\right\} \in \pi_{k+m-1}(\mathcal{G}) /\left(\operatorname{im} r_{k+m-1}\right)
$$

for each pair $f \in \operatorname{ker} r_{k-1}, f^{\prime} \in \pi_{m-1}\left(G_{p}\right)$ that is well defined modulo Samelson products of the form $\left\langle\beta, r \circ f^{\prime}\right\rangle, \beta \in \pi_{k}(\mathcal{G})$. The most important case is when $f$ is given by an inessential circle action. Lemma 2.5] states that rational homotopy of the group $S U(n)$ is generated by products of this form. In Section 3 we use characteristic classes to calculate $\left\{f, f^{\prime}\right\}$ in various other cases, for example when $f=f^{\prime}$ is given by an inessential Hamiltonian circle action.

The next proposition follows by combining Lemma 2.5 with the proof of Theorem 1.1. We shall state it in its most general form, ie, for a c-symplectic manifold $(M, a)$ and for the largest possible topological monoid $\mathcal{H}$. If $H^{1}(M ; \mathbb{R})=0$, we may take $\mathcal{H}$ to be the monoid $\mathcal{H}_{a}$ mentioned in Remark 1.2(iii). However, as we explain in more detail in Section 3 if $H^{1}(M ; \mathbb{R}) \neq 0$ then we must work in a context in which the $a$-Flux homomorphism Flux ${ }^{a}$ vanishes. Let $\mathcal{H}_{0}$ denote the identity component of $\mathcal{H}_{a}$, ie, the monoid formed by all smooth maps $M \rightarrow M$ that are homotopic to the identity. Then Flux ${ }^{a}: \pi_{1}\left(\mathcal{H}_{0}\right) \rightarrow H^{1}(M ; \mathbb{R})$ is given by

$$
\operatorname{Flux}^{a}(\lambda)(\gamma)=\left\langle a, \operatorname{tr}_{\lambda}(\gamma)\right\rangle \quad \text { for } \gamma \in H_{1}(M),
$$

where $\operatorname{tr}_{\lambda}(\gamma) \in H_{2}(M)$ is represented by $T^{2} \rightarrow M,(s, t) \mapsto \lambda(s)(\gamma(t))$. We shall denote by $\widetilde{\mathcal{H}}_{0}^{a}$ the cover of $\mathcal{H}_{0}$ determined by the $a$-Flux homomorphism. Since Flux ${ }^{a}$ is a homomorphism, $\widetilde{\mathcal{H}}_{0}^{a}$ is a monoid. Note also that in the symplectic case, $\operatorname{Flux}^{a}$ vanishes on all loops in $\operatorname{Ham}(M, \omega)$. Hence the map $g \mapsto\left(g,\left[g_{t}\right]\right)$ (where $\left\{g_{t}\right\}_{t \in[0,1]}$ is any path in Ham from the identity to $g$ ) defines an inclusion $\operatorname{Ham}(M, \omega) \rightarrow \widetilde{\mathcal{H}}_{0}^{a}$.

Proposition 1.4 Suppose that $G:=S U(2)$ acts smoothly and with finite kernel on a c-symplectic manifold $(M, a)$. Then the induced map

$$
\pi_{4}(B G) \rightarrow H_{4}\left(B \widetilde{\mathcal{H}}_{0}^{a}\right)
$$

is injective. Further, if $H^{1}(M)=0$ then the image of $\pi_{4}(B G)$ in $H_{4}\left(B \mathcal{H}_{a}\right)$ is nonzero.

If $(M, a)$ supports an action of $G:=S U(\ell)$ with $\ell>2$ then one can also try to understand the maps $\pi_{2 i}(B G) \otimes \mathbb{Q} \rightarrow H_{2 i}(B \mathcal{G})$ for $2<i \leq \ell$ and appropriate $\mathcal{G}$. Examples 4.1 and 4.2 show that these maps need not be injective. It is still possible that the injectivity of $R_{*}: \pi_{2 i}(B G) \otimes \mathbb{Q} \rightarrow \pi_{2 i}(B \mathcal{G}) \otimes \mathbb{Q}$ implies that of 
$h \circ R_{*}: \pi_{2 i}(B G) \otimes \mathbb{Q} \rightarrow H_{2 i}(B \mathcal{G})$, where $h$ denotes the rational Hurewicz map $\pi_{2 i}(B G) \otimes \mathbb{Q} \rightarrow H_{2 i}(B G) 5$

Here is one result in this direction. Again we denote by $\mathcal{H}_{0}:=\left(M^{M}\right)_{0}$, the space of (smooth) self maps of $M$ that are homotopic to the identity. Since the point evaluation map ev $: \pi_{*}(G) \rightarrow \pi_{*}(M)$ factors through $\pi_{*}\left(\mathcal{H}_{0}\right)$ the kernel of $r_{*}: \pi_{*}(G) \rightarrow \pi_{*}\left(\mathcal{H}_{0}\right)$ is contained in the kernel of $\mathrm{ev}_{*}$. We now describe conditions under which the kernel of the corresponding map

$$
h \circ R_{*}: \pi_{*}(B G) \otimes \mathbb{Q} \rightarrow H_{*}\left(B \mathcal{H}_{0}\right)
$$

on the classifying space level is contained in $\operatorname{ker} \mathrm{ev}_{*}$.

We say that $M$ satisfies the $c$-splitting condition for a topological monoid $\mathcal{H}$ that acts on $M$ if the associated (Hurewicz) fibration $M \rightarrow M_{\mathcal{H}} \rightarrow B \mathcal{H}$ is c-split, that is, if $\pi_{1}(B \mathcal{H})$ acts trivially on $H^{*}(M)$ and the Leray-Serre spectral sequence for rational cohomology degenerates at the $E_{2}$-term. For example, Blanchard showed in [6] that a simply connected Kähler manifold satisfies the c-splitting condition for $\mathcal{H}_{0}$ : see Lemma 4.4. Further, it was conjectured in 14 that any symplectic manifold satisfies the c-splitting condition for $\operatorname{Ham}(M, \omega)$. Recall that $M$ is called nilpotent if $\pi_{1}(M)$ is nilpotent and its action on $\pi_{*}(M) \otimes \mathbb{Q}$ is nilpotent [24]. The following result is proved in Section 4.2. For $\alpha \in \pi_{2 k-1} G$ we denote by $\bar{\alpha}$ the corresponding element in $\pi_{2 k}(B G)$.

Proposition 1.5 Let $G$ be a compact, connected, and simply connected Lie group that acts (with finite kernel) on a nilpotent manifold $M$. Suppose further that $M$ satisfies the $c$-splitting condition with respect to a monoid $\mathcal{H}$ containing $G$. Then for all $\alpha \in \pi_{*}(G)$

$$
\mathrm{ev}_{*}(\alpha) \neq 0 \text { in } \pi_{2 k-1}(M) \otimes \mathbb{Q} \Rightarrow h \circ R_{*}(\bar{\alpha}) \neq 0 \text { in } H_{2 k}(B \mathcal{H}) .
$$

In this proposition $M$ can be an arbitrary smooth manifold. However, if it is not c-symplectic there is no obvious way to define an interesting monoid $\mathcal{H}$ that satisfies the c-splitting condition. Proposition 4.6 is an alternative version with a weaker splitting condition that holds when $M$ is symplectic.

In the next results $\mathcal{H}_{H}$ denotes the submonoid of $\mathcal{H}$ that acts trivially on cohomology.

${ }^{5}$ Note that if $\mathcal{G}$ were a compact Lie group this would be obvious, since in this case all rational Whitehead products in $B \mathcal{G}$ vanish. However, it was shown in AbreuMcDuff [1] that Whitehead products do not always vanish when $\mathcal{G}:=\operatorname{Symp}(M, \omega)$. Hence in the generality considered here we cannot assume that $h$ is injective. 
Corollary 1.6 Consider the action of $G:=S U(\ell)$ on the manifold $M:=$ $G / T$ of complete flags, where $T$ is the maximal torus in $G$. Then the map $H_{*}(B G) \rightarrow H_{*}\left(B \mathcal{H}_{H}\right)$ is injective.

Proof It follows from Lemma 4.4 that $M$ satisfies the c-splitting condition for $\mathcal{H}_{H}$. Further $\mathrm{ev}_{*}: \pi_{*}(G) \otimes \mathbb{Q} \rightarrow \pi_{*}(M) \otimes \mathbb{Q}$ is injective. Hence $\pi_{*}(B G) \otimes \mathbb{Q} \rightarrow$ $H_{*}\left(B \mathcal{H}_{H}\right)$ is injective by Proposition 1.5. Since $H^{*}(B G)$ is freely generated by the duals of the spherical classes, the induced map $R^{*}: H^{*}(B \mathcal{H}) \rightarrow H^{*}(B G)$ is surjective, and so the corresponding map on rational homology is injective.

A general flag manifold can be written as

$$
M\left(m_{1}, \ldots, m_{k}\right):=U(\ell) / U\left(m_{1}\right) \times \cdots \times U\left(m_{k}\right), \quad m_{1} \geq \cdots \geq m_{k},
$$

where $\ell=\sum m_{i}$. In Proposition 4.8 below we extend the result of Corollary 1.6 to arbitrary flag manifolds. However the proof is considerably more complicated, and uses the characteristic classes defined in Section [3. As a warmup we give in Section 3 a new proof of the following extension of Reznikov's result about projective space.

Proposition 1.7 In the case of the action of $G=S U(n+1)$ on $M:=\mathbb{C P}^{n}$, the induced map $R_{*}: H_{*}(B G) \otimes \mathbb{Q} \rightarrow H_{*}\left(B \mathcal{H}_{H}\right)$ is injective.

The last section Section 5 investigates the image $\operatorname{ev}_{*}(\rho)$ of the element $\rho$ of Theorem 1.1 under the evaluation map, and shows its relation to certain Whitehead products in $\pi_{*}(M)$. For example, in Proposition 5.3 we give necessary and sufficient conditions for $\mathrm{ev}_{*}(\rho)$ to be nonzero, while Proposition 5.5 gives conditions under which the map $\mathrm{ev}_{*}: \pi_{5}(\mathcal{G}) \rightarrow \pi_{5}(M)$ is nonzero.

Acknowledgements We warmly thank the referees for making several important suggestions that have helped to simplify and clarify various arguments, in particular the proofs of Propositions 2.2 3.1 and 4.8. The first author thanks Thomas Vogel for discussions.

The first author is a member of EDGE, Research Training Network HPRN-CT2000-00101, supported by the European Human Potential Programme. He is also partly supported by the KBN grant 1P03A 02327 . The second author is partly supported by the NSF grant DMS 0305939. 


\section{A secondary product on homotopy groups of clas- sifying spaces}

If $\mathcal{G}$ is a topological group the Samelson product $\left\langle f, f^{\prime}\right\rangle \in \pi_{i+j}(\mathcal{G})$ of the elements $f \in \pi_{i}(\mathcal{G}), f^{\prime} \in \pi_{j}(\mathcal{G})$ is given by the map

$$
S^{i+j}=S^{i} \times S^{j} / S^{i} \vee S^{j} \rightarrow \mathcal{G}: \quad(x, y) \mapsto\left\langle f(x), f^{\prime}(y)\right\rangle .
$$

Here $\langle a, b\rangle$ denotes the commutator $a b a^{-1} b^{-1}$, and we take the base point of $\mathcal{G}$ to be the identity element so that the commutator vanishes on the wedge $S^{i} \vee S^{j}$. The main fact we shall use about this product is that (up to sign) it is the desuspension of the Whitehead product. Thus the obvious isomorphism $\pi_{*}(\mathcal{G}) \cong \pi_{*+1}(B \mathcal{G})$ takes $\pm\left\langle f, f^{\prime}\right\rangle$ to the Whitehead product $\left[F, F^{\prime}\right]$ of the images $F, F^{\prime}$ of $f, f^{\prime}$. Another important fact is that the Whitehead product vanishes rationally on $B G$ when $G$ is a compact connected Lie group. This follows from minimal model theory: nonzero Whitehead products lie in the kernel of the Hurewicz homomorphism and give rise to relations in rational cohomology, but $H^{*}(B G ; \mathbb{Q})$ is a free algebra. Hence the rational Samelson product also vanishes on Lie groups, but need not vanish when $\mathcal{G}$ is a symplectomorphism group.

All results in this section are concerned with rational homotopy and homology. One could therefore work in the rational homotopy category, in which case the notation $S^{k}$ does not denote a sphere but rather its image $S_{\mathbb{Q}}^{k}$ in this category. Alternatively, one can take sufficiently high multiples of all maps so that they are null homotopic rather than zero in $\pi_{*} \otimes \mathbb{Q}$, and then work in usual category. We adopt the latter approach.

\subsection{A general construction}

The first main result of this section is Proposition 2.1. In Corollary 2.3 we establish the uniqueness (but not the nontriviality) of the element $\rho$ of Theorem 1.1

Consider the sequence of fibrations associated with this action:

$$
\mathcal{G}_{p} \stackrel{i}{\longrightarrow} \mathcal{G} \stackrel{e v}{\longrightarrow} M \stackrel{j}{\longrightarrow} B \mathcal{G}_{p}=M_{\mathcal{G}} \stackrel{\pi}{\longrightarrow} B \mathcal{G},
$$

where $M_{\mathcal{G}}$ denotes the total space of the universal $M$-bundle over $B \mathcal{G}$.

Let $f: S^{k-1} \rightarrow \mathcal{G}_{p}$ and $f^{\prime}: S^{m-1} \rightarrow \mathcal{G}_{p}$ be maps satisfying the following assumptions: 
(A1) The Samelson product is trivial, $\left\langle f, f^{\prime}\right\rangle=0$;

(A2) The map $i \circ f$ is null homotopic in $\mathcal{G}$.

Equivalently, for the corresponding maps to the classifying space, $F: S^{k} \rightarrow B \mathcal{G}_{p}$ and $F^{\prime}: S^{m} \rightarrow B \mathcal{G}_{p}$ we have

(B1) The Whitehead product is trivial, $\left[F, F^{\prime}\right]=0$;

(B2) The map $\pi \circ F$ is null homotopic in $B \mathcal{G}$; in other words $F=j \circ \alpha$, where $\alpha: S^{k} \rightarrow M$.

These assumptions permit the construction of the following commutative diagram:

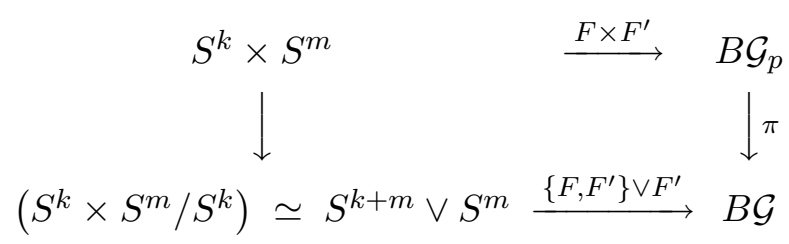

Here $F \times F^{\prime}$ is some extension of $F \vee F^{\prime}$, and exists because $\left[F, F^{\prime}\right]=0$. By (B2) this map descends to $S^{k} \times S^{m} / S^{k} \rightarrow B \mathcal{G}$. But $S^{k} \times S^{m} / S^{k} \simeq S^{m+k} \vee S^{m}$ because the attaching map of the top cell in the quotient $S^{k} \times S^{m} / S^{k}$ is null homotopic. (It is the Whitehead product of the trivial map on $S^{k}$ with the identity map of $S^{m}$.) We denote the homotopy class of the induced map on $S^{m+k}$ by $\left\{F, F^{\prime}\right\}$, and by $\left\{f, f^{\prime}\right\}$ the corresponding element of $\pi_{k+m-1}(\mathcal{G})$.

Note that the homotopy class $\left\{F, F^{\prime}\right\}$ may depend on the choice of extension of $F \vee F^{\prime}$ to $S^{k} \times S^{m}$ as well as on the chosen null homotopy $H_{t}, t \in[0,1]$, of $\pi \circ F$. Further it is not symmetric: indeed the conditions under which $\left\{F^{\prime}, F\right\}$ is defined are different from those for $\left\{F, F^{\prime}\right\}$.

This construction is a particular case of the secondary Whitehead product. For any pair of spaces $(X, A)$, let $\widehat{F} \in \pi_{k+1}(X, A), F^{\prime} \in \pi_{m}(A)$ be such that $\left[\partial \widehat{F}, F^{\prime}\right]=0 \in \pi_{m+k-1}(A)$. Consider the following diagram

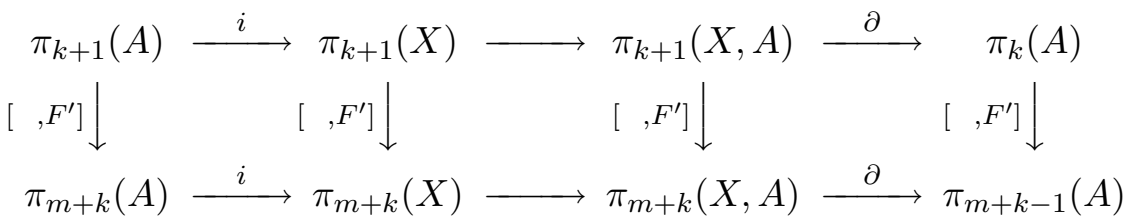

Then $\left[\widehat{F}, F^{\prime}\right] \in \pi_{m+k}(X, A)$ has trivial image in $\pi_{m+k-1}(A)$ and so lifts to $\pi_{m+k}(X)$. The secondary Whitehead product

$$
\left\{\widehat{F}, F^{\prime}\right\} \in \pi_{m+k}(X) / i\left(\pi_{m+k}(A)\right)
$$


is defined to be such a lift. In our situation $X=B \mathcal{G}$ and $A=B \mathcal{G}_{p}$. We are given two maps $F, F^{\prime} \in \pi_{*}(A)$ where $F$ has trivial image in $\pi_{*}(X)$. Therefore we must choose a lift $\widehat{F}$ of $F$ to $\pi_{k+1}(X, A)$ which adds an extra indeterminacy $\left[\pi_{k+1}(X), F^{\prime}\right]$ to the element $\left\{F, F^{\prime}\right\}:=\left\{\widehat{F}, F^{\prime}\right\}$.

We next specialize further to the case when $A=B G_{p}$, where $G_{p}$ is a Lie group. Then all Whitehead products in $\pi_{*}(A) \otimes \mathbb{Q}$ vanish, and we obtain the following result.

Proposition 2.1 Suppose that the maps $f, f^{\prime}$ take values in a Lie group $G_{p}$ that maps to $\mathcal{G}_{p}$ via $r$. Then for each $f \in \pi_{k-1}\left(G_{p}\right)$ such that $r \circ f$ is nullhomotopic in $\mathcal{G}$ the above construction gives a homomorphism

$$
\pi_{m-1}\left(G_{p}\right) \rightarrow \pi_{k+m}(B \mathcal{G}) \otimes \mathbb{Q} / \mathcal{K}, \quad f^{\prime} \mapsto\left\{F, F^{\prime}\right\}+\mathcal{K} .
$$

Here $\mathcal{K}$ is the subgroup of $\pi_{k+m}(B \mathcal{G}) \otimes \mathbb{Q}$ generated by $R_{*}\left(\pi_{k+m}\left(B G_{p}\right)\right)$ and the Whitehead products $\left[\beta, R \circ F^{\prime}\right]$, where $\beta \in \pi_{k+1}(B \mathcal{G})$ and $R: B G_{p} \rightarrow B \mathcal{G}$ is induced by $r$. In particular, if $r \circ f^{\prime}$ is also nullhomotopic in $\mathcal{G}$ then $\left\{F, F^{\prime}\right\}$ is well defined modulo the image of $\pi_{k+m}\left(B G_{p}\right)$. Further in this case $\left\{F^{\prime}, F\right\}$ is defined and equal to $\pm\left\{F, F^{\prime}\right\}$ modulo the image of $\pi_{k+m}\left(B G_{p}\right)$.

Proof The first two statements are an immediate consequence of the previous remarks. To see that $\left\{F^{\prime}, F\right\}= \pm\left\{F, F^{\prime}\right\}$ when $r \circ f$ and $r \circ f^{\prime}$ are both null homotopic, observe that the two relative Whitehead products

$$
\begin{array}{lll}
\left(D^{i+j}, S^{i+j-1}\right) & \stackrel{W}{\longrightarrow}\left(D^{i+1} \vee S^{j}, S^{i} \vee S^{j}\right) \\
\left(D^{i+j}, S^{i+j-1}\right) & \longrightarrow & \left(S^{i} \vee D^{j+1}, S^{i} \vee S^{j}\right)
\end{array}
$$

become homotopic in $\left(D^{i+1} \vee D^{j+1}, S^{i} \vee S^{j}\right)$ since takng the boundary gives an isomorphism $\pi_{i+j}\left(D^{i+1} \vee D^{j+1}, S^{i} \vee S^{j}\right) \rightarrow \pi_{i+j-1}\left(S^{i} \vee S^{j}\right)$. Hence one gets the same answer if one defines the relative product $\left\{F, F^{\prime}\right\}$ by using the nullhomotopy of either variable. The sign might change when one reverses the order.

We next give an explicit formula for $\left\{f, f^{\prime}\right\}$ under the assumptions (A1), (A2). Choose a map $c: D^{k+m-1} \rightarrow \mathcal{G}_{p}$ such that $\partial c=\left\langle f, f^{\prime}\right\rangle$ and an extension $\widetilde{f}: D^{k} \rightarrow \mathcal{G}$. Define an element $\beta=:\left\langle\widetilde{f}, f^{\prime}\right\rangle \in \pi_{k+m-1}(\mathcal{G})$ as follows.

$$
\begin{aligned}
& \beta:\left(D^{k} \times S^{m-1}\right) \cup D^{k+m-1} \longrightarrow \mathcal{G}, \\
& \beta(x, t)=\left\langle\widetilde{f}(x), f^{\prime}(t)\right\rangle \quad \text { for }(x, t) \in D^{k} \times S^{m-1} \\
& \beta(z)=c(z) \quad \text { for } z \in D^{k+m-1} \text {, }
\end{aligned}
$$


where $\langle\cdot, \cdot\rangle$ is the commutator as before. In what follows we shall assume that the maps $f, f^{\prime}$ take values in $G_{p}$ and we choose $c$ also to take values in $G_{p}$. Then $\beta$ is well defined modulo the image of $\pi_{k+m-1}\left(G_{p}\right)$ and Samelson products of the form $\left\langle\gamma, f^{\prime}\right\rangle$, where $\gamma \in \pi_{k}(\mathcal{G})$.

Proposition 2.2 Let $f, f^{\prime}$ be as in Proposition 2.1. Denote by $\mathcal{K}^{\prime}$ the subgroup of $\pi_{k+m-1}(\mathcal{G})$ generated by the image of $\pi_{k+m-1}\left(G_{p}\right)$ and Samelson products of the form $\left\langle\gamma, f^{\prime}\right\rangle$, where $\gamma \in \pi_{k}(\mathcal{G})$. Then, if we define $\beta=\left\langle\widetilde{f}, f^{\prime}\right\rangle$ by (2.2) taking $c$ also to have values in $G_{p}, \beta$ represents the element of $\left\{f, f^{\prime}\right\} \in \pi_{k+m-1}(\mathcal{G}) / \mathcal{K}^{\prime}$ corresponding to $\left\{F, F^{\prime}\right\}$ of Proposition 2.1] under the obvious isomorphism $\pi_{k+m-1}(\mathcal{G}) / \mathcal{K}^{\prime} \cong \pi_{k+m}(B \mathcal{G}) / \mathcal{K}$.

Before giving the proof we derive some easy corollaries.

Corollary 2.3 Let $\lambda: S^{1} \rightarrow \mathcal{G}_{p}$ be a circle action that is inessential in $\mathcal{G}$. Then $\{\lambda, \lambda\}$ is defined and equals the element $\rho \in \pi_{3}(\mathcal{G})$ given by (1.1). Further, it is independent of choices.

Proof Take $G=S^{1}$. Then it is immediate that $\{\lambda, \lambda\}$ is defined. It is independent of choices by Proposition 2.1 and the fact that $\pi_{3}(G)=0$. It equals $\rho$ by Proposition 2.2 .

Corollary 2.4 Suppose given a homomorphism $h: S^{1} \times K \rightarrow \mathcal{G}_{p}$, where $K$ is a Lie group. Let $\lambda:=\left.h\right|_{S^{1} \times\{1\}}$ and $f^{\prime} \in \pi_{m}(K)$. Suppose that $\lambda$ is inessential in $\mathcal{G}$. Then we may construct the element $\left\{\lambda, f^{\prime}\right\} \in \pi_{2+m}(\mathcal{G})$ so that it is well defined modulo the Samelson products $\left\langle\beta, f^{\prime}\right\rangle, \beta \in \pi_{2}(\mathcal{G})$.

Proof Define $\left\{\lambda, f^{\prime}\right\}$ using formula (2.2) noting that we may take $c$ to be constant since, by assumption, the maps $\lambda$ and $f^{\prime}$ commute. Then the only indeterminacy is of the form $\left\langle\beta, f^{\prime}\right\rangle, \beta \in \pi_{2}(\mathcal{G})$.

To show that $\{\lambda, \lambda\} \neq 0$ it suffices to show that $\{\Lambda, \Lambda\} \neq 0$, where $\Lambda: S^{2} \rightarrow$ $B S^{1}$ generates $\pi_{2}\left(B S^{1}\right)$ and hence that the composite map

$$
S^{2} \times S^{2} \longrightarrow B S^{1} \longrightarrow B \mathcal{G}_{p} \stackrel{\pi}{\longrightarrow} B \mathcal{G}
$$

is nontrivial on $H_{4}$. This will follow if we show that the map $B S^{1} \rightarrow B \mathcal{G}$ is nontrivial on $H_{4}$, which we do in Section 3 by evaluating some characteristic classes: see Corollary 3.7 
Proof of Proposition 2.2 One can prove this using the following commutative diagram that relates the relative Whitehead product to the relative Samelson product:

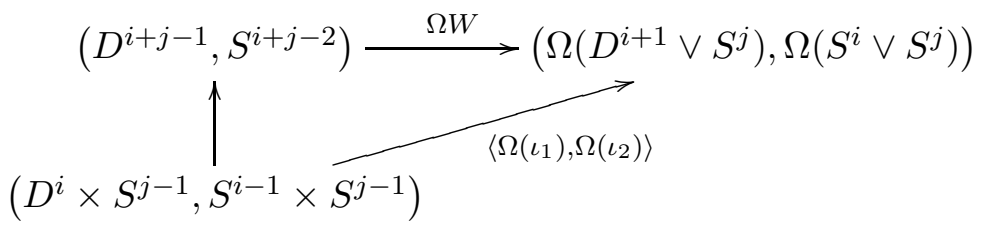

As above, $W$ is the universal model for the relative Whitehead product with looping $\Omega W$ and the left hand vertical map is induced by collapsing the spheres of dimensions $i-1$ and $j-1$. Further $\left\langle\Omega\left(\iota_{1}\right), \Omega\left(\iota_{2}\right)\right\rangle$ is the Samelson product of the maps obtained by looping the inclusions on each factor.

For readers who are unfamiliar with such homotopy theoretic arguments we now give a more explicit proof that describes the relevant homotopies in more detail. We begin by describing the map $\left\{F, F^{\prime}\right\}: S^{k+m} \rightarrow B \mathcal{G}$.

Consider $S^{k+m}$ as the union of three pieces $\mathcal{D}_{1} \cup \mathcal{D}_{0} \cup \mathcal{D}_{-1}$ where:

(a) $\mathcal{D}_{1}$ is the interior of the top cell in $S^{k} \times S^{m}$, and $\left.\left\{F, F^{\prime}\right\}\right|_{\mathcal{D}_{1}}$ equals $F \times F^{\prime}$;

(b) $\mathcal{D}_{0}$ is a cylinder $S^{k+m-1} \times[0,1]$ which is mapped by the composite

$$
S^{k+m-1} \times[0,1] \stackrel{e \times i d}{\longrightarrow}\left(S^{k} \vee S^{m}\right) \times[0,1] \stackrel{H_{t} \vee F^{\prime}}{\longrightarrow} B \mathcal{G},
$$

where $e: S^{k+m-1} \rightarrow S^{k} \vee S^{m}$ is the attaching map of the top cell in $S^{k} \times S^{m}$, and $H_{t}$ is a homotopy from $H_{1}=\pi \circ F$ to the constant map $H_{0}$; and

(c) $\mathcal{D}_{-1}$ is a capping disc, mapped by a fixed homotopy from $\left(H_{0} \vee F^{\prime}\right) \circ e$ to the constant map. This homotopy exists because the Whitehead product $\left[H_{0}, F^{\prime}\right]$ vanishes. Since $F^{\prime}\left(S^{m}\right) \subset B G_{p}$ we may assume that the restriction of $\left\{F, F^{\prime}\right\}$ to $\mathcal{D}_{-1}$ factors through a map $\mathcal{D}_{-1} \rightarrow B G_{p}$. Thus here we think of the domain as

$$
S^{k+m}=S^{k+m-1} \times[-1,2] / \sim,
$$

where the equivalence relation $\sim$ collapses each sphere

$$
S^{k+m-1} \times\{-1\}, \quad S^{k+m-1} \times\{2\}
$$

to a single point and we identify $\mathcal{D}_{i}$ with $S^{k+m-1} \times[i, i+1]$.

Now choose any model for $B \mathcal{G}$ such that the induced map $B G_{p} \rightarrow B \mathcal{G}$ is injective, and denote by $E \mathcal{G}$ the space of paths in $B \mathcal{G}$ with fixed initial point. The map $\Psi: S^{k+m} \rightarrow \mathcal{G}$ lifts to

$$
\widetilde{\Psi}: D^{k+m}:=D^{k+m-1} \times[-1,2] / \sim \longrightarrow E \mathcal{G} .
$$


We must show how to construct $\Psi$ so that the restriction of $\widetilde{\Psi}$ to the boundary

$$
S^{k+m-1}:=\partial D^{k+m-1} \times[-1,2] / \sim
$$

agrees with formula (2.2). To do this we construct $\Psi$ using data coming from $\mathcal{G}$ rather than $B \mathcal{G}$. Again, we think of $S^{k+m-1}$ as divided into three pieces, a central cylinder $\mathcal{C}_{0}:=\partial D^{k+m-1} \times[0,1]$ together with two capping $\operatorname{discs} \mathcal{C}_{-1}, \mathcal{C}_{1}$.

Choose the null homotopy $H_{t}: S^{k} \rightarrow B \mathcal{G}, t \in[0,1]$, to be the image under suspension of the null homotopy $h_{t}: S^{k-1} \rightarrow \mathcal{G}$, where $h_{t}$ is the restriction of $\widetilde{f}$ to the sphere of radius $t$ in $D^{k}$. Then the Whitehead product $\left[H_{t}, F^{\prime}\right]$ desuspends to the Samelson product $\left\langle h_{t}, f^{\prime}\right\rangle$ for each $t \in[0,1]$. Hence we may define $\Psi$ on the cylinder $\mathcal{D}_{0}$ to have a lift $\widetilde{\Psi}$ that restricts on $\mathcal{C}_{0}:=$ $\partial D^{k+m-1} \times[0,1]$ to the family of maps $\left\langle h_{t}, f^{\prime}\right\rangle$ defined as in (2.1).

When $t=0,1$ the Samelson products $\left\langle h_{t}, f^{\prime}\right\rangle$ lie in $G_{p}$ and we may choose $\Psi$ and its lift $\widetilde{\Psi}$ so that $\widetilde{\Psi}$ maps the discs $D^{k+m-1} \times\{0,1\}$ into $E G_{p}$. Moreover, $\left\langle h_{0}, f^{\prime}\right\rangle$ is constant, since $h_{0}$ is the constant map at the identity element. Thus the restriction of $\widetilde{\Psi}$ to $D^{k+m-1} \times\{0\}$ descends to a map $D^{k+m-1} / \partial=$ $S^{k+m-1} \rightarrow E G_{p}$. Then $\widetilde{\Psi}$ extends to the ball $D^{k+m}$ because $E G_{p}$ is contractible, and we define $\Psi$ on $\mathcal{D}_{-1} \equiv D^{k+m}$ to be the projection of $\widetilde{\Psi}$ to $B G_{p}$ followed by the map $B G_{p} \rightarrow B \mathcal{G}$.

Finally consider the disc $\mathcal{D}_{1}$. The chosen null homotopy $c$ of $\left\langle f, f^{\prime}\right\rangle$ defines a map of the $\operatorname{disc} D^{\prime}=\partial D^{k+m-1} \times[1,2] / \sim$ to $G_{p}$ that agrees with the restriction of $\widetilde{\Psi}$ to $\partial D^{\prime}=\partial D^{k+m-1} \times\{1\}$. This gives a map of the $(k+m)$-sphere $D^{\prime} \cup\left(D^{k+m-1} \times\{1\}\right)$ into the contractible space $E G_{p}$. Again, choose $\widetilde{\Psi}$ on the disc $D^{k+m-1} \times[1,2] / \sim$ to be any extension and define $\left.\Psi\right|_{\mathcal{D}_{1}}$ to be the corresponding map to $B \mathcal{G}$.

\subsection{Calculations in $S U(n+1)$}

We end this section by further investigating the construction in Corollary 2.4 in the case when the homomorphism $h$ takes values in a Lie group $H_{p}$. Thus we suppose that $h: S^{1} \times K \rightarrow H_{p}$. For simplicity we restrict to the case when $H$ is a subgroup of $G:=S U(n+1)$ and $H_{p}$ is contained in $G_{p}:=U(n)$. (Thus $G / G_{p}=\mathbb{C P}^{n}$.) Unless explicit mention is made to the contrary, we embed $S U(k)$ in $S U(k+1)$ as the subgroup that acts on the first $k$ coordinates, and similarly for inclusions $U(k) \subset U(k+1)$.

For $k=1, \ldots, n$ consider the commuting circle actions

$$
\lambda_{k}: S^{1} \rightarrow S U(k+1) \subset S U(n)
$$


where $\lambda_{1}$ has weights $(1,-1,0 \ldots, 0), \lambda_{2}$ has weights $(1,1,-2,0, \ldots, 0)$ and $\lambda_{k}$ has weights $(1, \ldots, 1,-k, 0, \ldots, 0)$. Since $\lambda_{1}$ contracts in $S U(2)$ and $\pi_{2}(U(k))$ $=0$, the construction of Corollary 2.4 gives a well defined element

$$
\alpha_{3}:=\left\{\lambda_{1}, \lambda_{1}\right\} \in \pi_{3}(S U(2)) .
$$

Because $\lambda_{2}$ commutes with $S U(2)$ and contracts in $S U(3)$ we may repeat to get a well defined element

$$
\alpha_{5}:=\left\{\lambda_{2}, \alpha_{3}\right\}=\left\langle\widetilde{\lambda}_{2}, \alpha_{3}\right\rangle \in \pi_{5}(S U(3)),
$$

where $\widetilde{\lambda}_{2}$ is a contraction of $\lambda_{2}$ and we use formula (2.2). This is a version of the construction in Corollary 2.4. To see this in general, define the homomorphism $h_{k}: S^{1} \times U(k) \rightarrow U(k)$ to be the identity on the second factor and to take $U(1)$ to the center of $U(k)$ in the obvious way, and define $\iota_{k}: U(k) \rightarrow S U(k+1)$ by

$$
\iota_{k}(A)=\left(\begin{array}{cc}
A & 0 \\
0 & (\operatorname{det} A)^{-1}
\end{array}\right) .
$$

Then the composite $\iota_{k} \circ h_{k}: S^{1} \rightarrow S U(k+1)$ is precisely $\lambda_{k}$, and assuming that $\alpha_{2 k-1} \in \pi_{2 k-1}(S U(k))$ is already defined we may set:

$$
\alpha_{2 k+1}:=\left\{\lambda_{k}, \alpha_{2 k-1}\right\}=\left\langle\widetilde{\lambda}_{k}, \alpha_{2 k_{1}}\right\rangle \in \pi_{2 k+1}(S U(k+1)),
$$

where $\widetilde{\lambda}_{k}: D^{2} \rightarrow S U(k+1)$ is a contraction of $\lambda_{k}$.

Lemma $2.5 \alpha_{2 k+1} \in \pi_{2 k+1}(S U(k+1))$ is nonzero for $k=1, \ldots n$.

Proof Let $\mathbb{T}^{n}$ be the diagonal torus in $S U(n+1)$ and denote by $\Lambda_{k}: S^{2} \rightarrow$ $B \mathbb{T}^{k}$ the desuspension of $\lambda_{k}$. Because Whitehead products vanish in $B \mathbb{T}^{k}$, the map $\Lambda_{1} \vee \Lambda_{1} \vee \Lambda_{2} \vee \cdots \vee \Lambda_{k}: \vee_{k+1} S^{2} \rightarrow B \mathbb{T}^{k}$ has an extension to

$$
\prod_{i=1}^{k+1} S^{2} \stackrel{g_{k}=\Lambda_{1} \times \Lambda_{1} \times \Lambda_{2} \times \cdots \times \Lambda_{k}}{\longrightarrow} B \mathbb{T}^{k}
$$

that is unique up to homotopy. Taking the composite with the inclusion $B \mathbb{T}^{k} \rightarrow$ $B U(k+1)$ gives a rank $(k+1)$ bundle over $\prod S^{2}$. We show below that it suffices to show that this bundle has some nontrivial Chern classes in dimension $2 k+2$. We then calculate the pullback of $c_{k+1}$.

We first claim that for each $k \in\{1, \ldots, n\}$ there is a homotopy commutative diagram

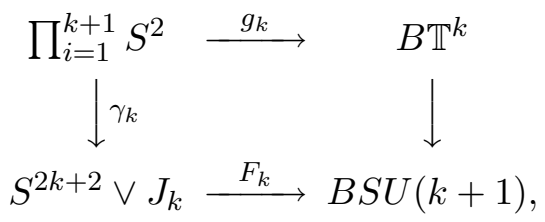


in which the CW complex $J_{k}$ has dimension $\leq 2 k$. We construct this diagram by induction on $k$. The only difficulty is to construct the left vertical map $\gamma_{k}$. When $k=1$ the diagram reduces to

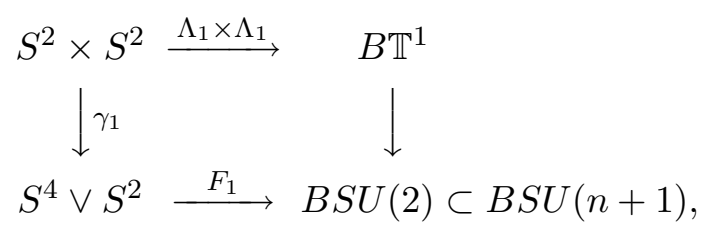

and is a special case of the situation discussed before Proposition 2.1. For $k>1$ we may assume by induction that the map $\prod_{i=1}^{k+1} S^{2} \rightarrow B S U(k+1)$ given by going horizontally to $B \mathbb{T}^{k}$ and then vertically to $B S U(k+1)$ factors through

$$
S^{2} \times\left(S^{2 k} \vee J_{k}\right) \stackrel{\Lambda_{k} \times F_{k-1}}{\longrightarrow} B Z_{k} \times B S U(k) \rightarrow B S U(k+1) \subset B S U(n+1),
$$

where $Z_{k}$ denotes the commutator of $S U(k)$ in $S U(k+1)$. Since $\Lambda_{k}$ contracts in $B S U(k+1)$ the product $\Lambda_{k} \times F_{k-1}$ is homotopic via maps to $B S U(k+1)$ to a map that takes the sphere $S^{2} \times\{p t\}$ to a single point. But when one contracts the first sphere in the product $S^{2} \times\left(S^{2 k} \vee J_{k-1}\right)$ one obtains a space of the form $S^{2 k+2} \vee J_{k}$. Thus the above diagram exists for all $k$.

Next observe that $g_{k}$ factors through the inclusion $B U(k) \rightarrow B S U(k+1)$ given by

$$
A \mapsto\left(\begin{array}{cc}
A & 0 \\
0 & \operatorname{det} A^{-1}
\end{array}\right)
$$

Therefore we may apply Proposition 2.2 to conclude that the restriction of $F_{k}$ to the top sphere $S^{2 k+2}$ desuspends to $\alpha_{2 k+1} \in \pi_{2 k+1} S U(k+1)$ ) modulo the subgroup $\mathcal{K}^{\prime}$ generated by the image of $\pi_{2 k+1}(U(k))$ and certain Samelson products. Since $\mathcal{K}^{\prime}=0$, we find that $\alpha_{2 k+1} \neq 0$ if and only if $\left.F_{k}\right|_{S^{2 k+2}}$ is nonzero. This will be the case precisely when the bundle represented by the composite

$$
\prod S^{2} \stackrel{g_{k}}{\longrightarrow} B \mathbb{T}^{k} \longrightarrow B S U(k+1)
$$

has nontrivial top dimensional Chern classes.

We now check this by calculating the pullback of $c_{k+1}$. 6 Denote by $\mathbb{T}:=$ $\left(S^{1}\right)^{k+1}$ the diagonal subgroup in $U(k+1)$ and by $t_{1}, \ldots, t_{k+1} \in H^{2}(B \mathbb{T})$ the obvious generators of $H^{*}(B \mathbb{T})$. Then $c_{k+1}$ pulls back to $t_{1} \ldots t_{k+1} \in$

\footnotetext{
${ }^{6}$ Because the map $B S U(k+1) \rightarrow B U(\infty)$ is $(2 k+2)$-connected, one can equally well phrase this calculation in terms of the structure of the induced stable bundle on $\prod S^{2}$.
} 
$H^{2 k+2}(B \mathbb{T})$. The map $B \mathbb{T}^{k} \rightarrow B U(k+1)$ factors through $B \mathbb{T}$ and it suffices to show that the pull back of $t_{1} \ldots t_{k+1}$ by the composite

$$
\Phi: \prod S^{2} \stackrel{g_{k}}{\longrightarrow} B \mathbb{T}^{k} \longrightarrow B \mathbb{T}
$$

does not vanish. For $j=0,1, \ldots, k$ denote by $y_{j}$ the pullback to $\prod_{j=0}^{k} S^{2}$ of the generator of $H^{2}\left(S^{2}\right)$ by the projection onto the sphere that is mapped by $\Lambda_{j}$ (where we set $\Lambda_{0}:=\Lambda_{1}$.) Then

$$
\begin{aligned}
& \Phi^{*}\left(t_{1} \ldots t_{k+1}\right)=\left(y_{0}+y_{1}+y_{2}+\cdots+y_{k}\right)\left(-y_{0}-y_{1}+y_{2}+\cdots+y_{k}\right) \times \\
& \times \prod_{j=2}^{k}\left(-j y_{j}+\sum_{i>j} y_{i}\right) \\
&=2(-1)^{k} k ! y_{0} y_{1} y_{2} \ldots y_{k} .
\end{aligned}
$$

since $y_{j}^{2}=0$ for all $j$. Since this is nonzero, the proof is complete.

Remark 2.6 One can construct a nontrivial element in $\pi_{2 k+2}(B S U(k+2))$ for $k \geq 1$ by the following inductive procedure. Let $E_{2} \rightarrow S^{2}$ be the complex line bundle classified by the map $\Lambda_{2}: S^{2} \rightarrow B U(1)$. Note that its first Chern class is equal to the generator $y \in H^{2}\left(S^{2}\right)$. Suppose also that we have already constructed a map $\Lambda_{2 k}: S^{2 k} \rightarrow B U(k)$ that classifies a bundle $E_{2 k}$ with nontrivial Chern class $c_{k}\left(E_{2 k}\right)$. Then construct a homotopy commutative diagram

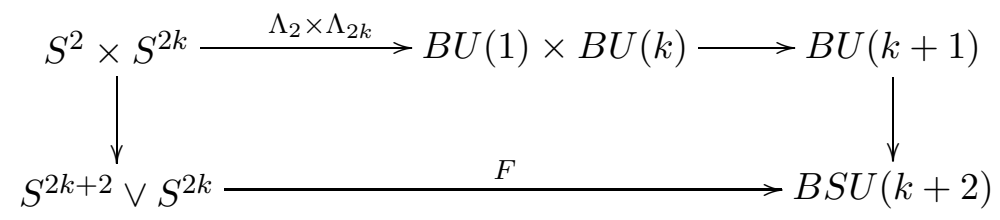

in which the top row classifies the bundle $E_{2} \times E_{2 k} \rightarrow S^{2} \times S^{2 k}$ whose Chern class $c_{k+1}=p_{1}^{*} c_{1}\left(E_{2}\right) \cup p_{2}^{*} c_{k}\left(E_{2 k}\right) \neq 0$. Since $\Lambda_{2}$ contracts in $B S U(k+1)$ the bottom row of the diagram is constructed in the usual way, and the induced map $S^{2 k+2} \rightarrow B S U(k+2)$ is homotopically nontrivial. But note that it does not desuspend to the element of $\pi_{2 k+1}(S U(k+2))$ constructed via commutators as in Corollary 2.4. For one can choose the contraction of $\Lambda_{2}$ to lie in a copy of $S U(2) \subset S U(k+2)$ that commutes with the image of $U(k)$. This is not a contradiction since Proposition 2.2 states only that the induced map should be given by (2.2) modulo elements of $\mathcal{K}^{\prime}$. But now $\mathcal{K}^{\prime}$ is the whole of $\pi_{2 k+1}(S U(k+$ 2)) since it includes the image of $\pi_{2 k+1}(U(k+1))$. 


\section{Characteristic classes}

Let $\mathcal{G}=\operatorname{Ham}(M, \omega)$ and consider the universal bundle $M \stackrel{j}{\rightarrow} M_{\mathcal{G}} \stackrel{\pi}{\rightarrow} B \mathcal{G}$. There is a unique class $[\Omega] \in H^{2}\left(M_{\mathcal{G}} ; \mathbb{R}\right)$ called the coupling class that extends the fiberwise symplectic class $[\omega]$ and has the property that the fiberwise integral $\int_{M}[\Omega]^{n+1} \in H^{2}(B \mathcal{G} ; \mathbb{R})$ vanishes. Following Januszkiewicz-Kȩdra [13], we define the classes

$$
\mu_{k}:=\int_{M}[\Omega]^{n+k} \in H^{2 k}(B \mathcal{G}):=H^{2 k}(B \mathcal{G} ; \mathbb{R}) .
$$

In this section we first generalize these classes to other groups and monoids, and then discuss computations and applications. When $H^{1}(M) \neq 0$ we shall work only with connected groups and monoids. The issues that arise in the general case are discussed from different perspectives in Gal-Kedra [7] and McDuff [17.

\subsection{The classes $\mu_{k}$}

Our first aim is to define the classes $\mu_{k}$ in as general a context as possible. Thus if $\mathcal{H}$ is a topological monoid that acts on a c-symplectic manifold $(M, a)$ we need to determine conditions on $\mathcal{H}$ that guarantee that the class $a \in H^{2}(M)$ has a well defined extension to a class $\widetilde{a} \in H^{2}\left(M_{\mathcal{H}}\right)$. As before we denote by $\mathcal{H}_{0}$ the identity component of the space of smooth maps $M \rightarrow M$ and by $\mathcal{H}_{a}$ the space of smooth homotopy equivalences that fix $a$. Further $M_{\mathcal{H}}$ denotes the total space of the universal $M$-bundle over $B \mathcal{H}$. Thus the projection $\pi: M_{\mathcal{H}} \rightarrow B \mathcal{H}$ is a Hurewicz fibration.

The $a$-Flux homomorphism Flux ${ }^{a}: \pi_{1}\left(\mathcal{H}_{0}\right) \rightarrow H^{1}(M ; \mathbb{R})$ was defined in equation (1.2). In the symplectic case the image

$$
\Gamma_{\omega}:=\operatorname{Flux}^{a}\left(\pi_{1}\left(\operatorname{Symp}_{0}\right)\right) \subset H^{1}(M ; \mathbb{R})
$$

is called the flux group, and there is a surjective homomorphism

$$
\operatorname{Flux}_{\omega}: \operatorname{Symp}_{0}(M, \omega) \rightarrow H^{1}(M ; \mathbb{R}) / \Gamma_{\omega}, \quad g \mapsto \int_{0}^{1}\left[\omega\left(\dot{g}_{t}, \cdot\right)\right] d t,
$$

where $g_{t}$ is any path in the connected group $\operatorname{Symp}_{0}(M, \omega)$ from the identity to $g$. The kernel of Flux $\omega$ is precisely the Hamiltonian group. In the c-symplectic case one cannot define a flux homomorphism on $\operatorname{Diff}_{0}(M)$ (which is a simple group), and the analog of the Hamiltonian group is the covering group

$$
\operatorname{Ham}(M, a)
$$


of $\operatorname{Diff}_{0}(M)$ corresponding to the kernel of Flux ${ }^{a}: \pi_{1}\left(\right.$ Diff $\left._{0}\right) \rightarrow H^{1}(M ; \mathbb{R})$. Thus there is an exact sequence of topological monoids

$$
\Gamma_{a} \rightarrow \operatorname{Ham}(M, a) \rightarrow \operatorname{Diff}_{0} M,
$$

where the fiber $\Gamma_{a}:=\operatorname{im}\left(\right.$ Flux $\left.^{a}\right)$ is given the discrete topology. 7 Note that in the symplectic case, $\operatorname{Ham}(M, \omega)$ is homotopy equivalent to the corresponding covering group of $\operatorname{Symp}_{0}(M, \omega)$. Further there is an inclusion $\operatorname{Ham}(M, \omega) \rightarrow$ $\operatorname{Ham}(M, a)$ given by taking the element $f \in \operatorname{Ham}(M, \omega)$ to the pair $\left[f,\left\{f_{t}\right\}\right]$, where $f_{t}, t \in[0,1]$, is any path in the connected $\operatorname{group} \operatorname{Ham}(M, \omega)$ from the identity to $f$. (Since two such paths differ by a loop with zero $a$-flux, this recipe defines a unique element $\left[f,\left\{f_{t}\right\}\right] \in \operatorname{Ham}(M, a)$.) Sometimes we will also work with the covering space $\widetilde{\mathcal{H}}_{0}^{a}$ of $\mathcal{H}_{0}$ corresponding to the kernel of Flux ${ }^{a}$. This is a topological monoid.

A smooth circle action on a c-symplectic manifold $(M, a)$ is said to be $c$ Hamiltonian if it is in the kernel of Flux ${ }^{a}$. As shown by Allday [2] such actions do have certain geometric properties; for example their fixed point set has at least two connected components, though these components need not be c-symplectic as would happen in the symplectic case.

Proposition 3.1 (i) Given $a \in H^{2}(M)$ such that $\int_{M} a^{n} \neq 0$, consider the fibration $M \rightarrow M_{\mathcal{H}} \rightarrow B \mathcal{H}$ where $\mathcal{H}:=\widetilde{\mathcal{H}}_{0}^{a}$. Then there is a unique element $\widetilde{a} \in H^{2}\left(M_{\mathcal{H}}\right)$ that restricts to $a \in H^{2}(M)$ and is such that $\int_{M} \widetilde{a}^{n+1}=0$.

(ii) If $H^{1}(M)=0$, such a class $\tilde{a}$ exists on $M_{\mathcal{H}_{a}}$.

The above class $\widetilde{a}$ is called the coupling class.

Proof (i) Consider the Leray-Serre cohomology spectral sequence for the (Hurewicz) fibration $M \rightarrow M_{\mathcal{H}} \rightarrow B \mathcal{H}$. Since $\mathcal{H}$ is connected the $E_{2}$-term is a product and the class $a$ lies in $E_{2}^{2,0}=H^{2}(M) \otimes H^{0}(B \mathcal{H})$. As explained for example in [14, Lemma 2.2] the differential

$$
d_{2}: E_{2}^{2,0} \longrightarrow E_{2}^{1,2}=H^{1}(M) \otimes H^{2}(B \mathcal{H})
$$

is determined by the flux homomorphism. More precisely, if we consider the elements of $H^{1}(M) \otimes H^{2}(B \mathcal{H})$ as homomorphisms $H_{2}(B \mathcal{H}) \rightarrow H^{1}(M)$, then

$$
d_{2}(a)(\bar{\lambda})=\operatorname{Flux}^{a}(\lambda),
$$

\footnotetext{
${ }^{7}$ Ono 21 recently proved that the symplectic flux group $\Gamma_{\omega}$ is a discrete subroup of $H^{1}(M ; \mathbb{R})$. But $\Gamma_{a}$ need not be: see McDuff 15 .
} 
where $\bar{\lambda} \in \pi_{2}(B \mathcal{H}) \cong H_{2}(B \mathcal{H})$ corresponds to the loop $\lambda \in \pi_{1}(\mathcal{H})$. Hence, because $\lambda$ is c-Hamiltonian, $d_{2}(a)=0$. But the image of $a$ under $d_{3}: E_{3}^{2,0} \rightarrow$ $E_{3}^{0,3}$ must vanish: since $a^{n+1}=0$ in $H^{*}(M)$,

$$
0=d_{3}\left(a^{n+1}\right)=(n+1) a^{n} \otimes d_{3}(a) \in H^{2 n}(M) \otimes H^{3}(B \mathcal{H}),
$$

which is possible only if $d_{3}(a)=0$. Therefore $a$ survives into the $E_{\infty}$-term of the spectral sequence, and so has some extension $u \in H^{2}\left(M_{\mathcal{H}}\right)$.

To prove uniqueness note that because $B \mathcal{H}$ is simply connected the kernel of the restriction map $H^{2}\left(M_{\mathcal{H}}\right) \rightarrow H^{2}(M)$ is isomorphic to the pullback of $H^{2}(B \mathcal{H})$. A short calculation shows that we may take

$$
\widetilde{a}:=u-\frac{1}{n+1} \pi^{*} \pi_{!}\left(u^{n+1}\right),
$$

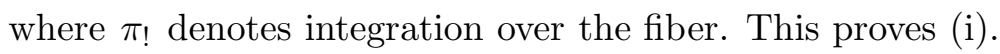

Now consider (ii). Because the elements in $\mathcal{H}:=\mathcal{H}_{a}$ preserve $a$ there is an element $a$ in the $E_{2}^{0,2}$-term in the Leray-Serre spectral sequence for the Hurewicz fibration $M_{\mathcal{H}_{a}} \rightarrow B \mathcal{H}_{a}$. Since $H^{1}(M)=0$ the differential $d_{2}$ must vanish and the argument showing that $d_{3}(a)=0$ still holds. Further the uniqueness proof goes through as before: although $B \mathcal{H}$ need no longer be simply connected, the fact that $H^{1}(M)=0$ implies that $E_{*}^{1,1}=0$. Hence the kernel of the restriction map $H^{2}\left(M_{\mathcal{H}}\right) \rightarrow H^{2}(M)$ is still isomorphic to the pullback of $H^{2}(B \mathcal{H})$. This proves (ii).

Remark 3.2 (i) If the first Chern class of $(M, \omega)$ is a nonzero multiple of the symplectic class $a$, then there is an easier way to find an extension $u \in H^{2}\left(M_{\mathcal{H}}\right)$ of the symplectic class: simply take it to be an appropriate multiple of the first Chern class of the vertical tangent bundle. This construction applies whenever $\mathcal{H}$ acts in such a way that the vertical bundle has a complex structure. The most natural choice for $\mathcal{H}$ is the group of symplectomorphisms. Note that we cannot take it to be the diffeomorphism group.

(ii) Let $(M, a)$ be any c-symplectic manifold and consider an $M$-bundle $P \rightarrow$ $B$. We saw above that if $a$ survives into the $E_{2}$ term of the Leray-Serre spectral sequence then $d_{3}(a)=0$ and $a$ survives to $E_{\infty}$. This shows that the obstruction to the existence of an extension $\widetilde{a}$ of $a$ depends only on the restriction of $P \rightarrow B$ over the 2 -skeleton of $B$.

Definition 3.3 Let $(M, a)$ be a c-symplectic manifold and let $\mathcal{H}$ denote either the monoid $\widetilde{\mathcal{H}}_{0}^{a}$ or, if $H^{1}(M)=0$, the monoid $\mathcal{H}_{a}$. We define $\mu_{k} \in H^{2 k}(B \mathcal{H})$ by

$$
\mu_{k}:=\pi_{!}\left(\widetilde{a}^{n+k}\right),
$$


where $\widetilde{a}$ is the coupling class constructed in Proposition 3.1

These classes $\mu_{k}$ extend those defined in equation (3.1). Note that $\mu_{1} \equiv 0$ by definition. We will see in the next section that the pullback of $\mu_{2 k}$ to $H^{*}\left(B S^{1}\right)$ is nonzero for all $k>0$ and for every nontrivial c-Hamiltonian circle action on $M$.

Proof of Proposition 1.7 We must show that if $G=S U(n+1)$ acts on $M:=\mathbb{C P}^{n}$ then the induced map $R_{*}: \pi_{*}(B S U(n+1) \otimes \mathbb{Q}) \rightarrow H_{*}(B \mathcal{H})$ is injective where $\mathcal{H}:=\mathcal{H}_{a}$. For $2 \leq k \leq n+1$ choose $s_{2 k}: S^{2 k} \rightarrow B S U(n+1)$ so that $\alpha_{k}:=s_{2 k}^{*}\left(c_{k}\right)$ generates $H^{2 k}\left(S^{2 k}\right)$, where $c_{k}$ is the $k$ th Chern class. Consider the bundle $\mathbb{C P}^{n} \rightarrow P \stackrel{p}{\rightarrow} S^{2 k}$ associated with this element. (This is simply the projectivization of the corresponding rank $(n+1)$ vector bundle $E \rightarrow S^{2 k}$.) The Leray-Hirsch theorem states that the cohomology of $P$ is a free $H^{*}\left(S^{2 k}\right)$-module generated by the powers $1=c^{0}, \ldots, c^{n}$ of the first Chern class $c$ of the tautological line bundle. Since $2 \leq k \leq n+1$ there is $\beta_{k} \in H^{2 k}\left(S^{2 k}\right)$ so that

$$
c^{n+1}=p^{*}\left(\beta_{k}\right) \cup c^{n+1-k} .
$$

The theory of characteristic classes implies that $\beta_{k}=c_{k}(E)=s_{2 k}^{*}\left(c_{k}\right)=: \alpha_{k}$. Multiplying this equality by $c^{k-1}$ we obtain

$$
c^{n+k}=p^{*}\left(\alpha_{k}\right) \cup c^{n},
$$

which implies that $p_{!}\left(c^{n+k}\right)=$ const. $\alpha_{k} \neq 0$. Now observe that because $k \geq 2$, $H^{2}(P)$ has dimension 1 , so that $c$ equals the coupling class $\widetilde{a} \in H^{2}(P)$ up to a nonzero constant. Hence

$$
\left\langle\mu_{k}, R_{*}\left(\left[S^{2 k}\right]\right)\right\rangle=\text { const. }\left\langle\alpha_{k},\left[S^{2 k}\right]\right\rangle \neq 0 .
$$

Therefore $\pi_{*}(B G) \rightarrow H_{*}(B \mathcal{H})$ is injective. The conclusion now follows as in the proof of Corollary 1.6 .

Remark 3.4 Let $\mathcal{G}:=\operatorname{Ham}(M, a)$ and denote by $\mathcal{G}_{p}$ the homotopy fiber of the evaluation map $\mathcal{G} \rightarrow M$. Thus if the elements of $\mathcal{G}$ are pairs $\left(g,\left\{g_{t}\right\}\right)$ where $\left\{g_{t}\right\}$ is an equivalence class of paths from $i d$ to $g$, then $\mathcal{G}_{p}$ is the subgroup of $\mathcal{G}$ consisting of all pairs such that $g(p)=p$. The restriction $M_{\mathcal{G}_{p}} \rightarrow B \mathcal{G}_{p}$ of the universal bundle $M_{\mathcal{G}} \rightarrow B \mathcal{G}$ to $B \mathcal{G}_{p} \subset B \mathcal{G}$ has a canonical section $\sigma: B \mathcal{G}_{p} \rightarrow M_{\mathcal{G}_{p}}$ whose image $\sigma(b)$ at $b \in B \mathcal{G}_{p}$ is the point in the fiber $M_{b}$ corresponding to $p$. Thus there is a homotopy commutative diagram

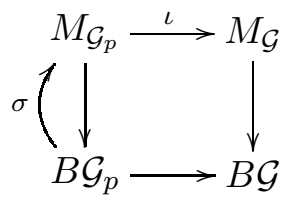


such that the composite $\iota \circ \sigma: B \mathcal{G}_{p} \rightarrow M_{\mathcal{G}}$ is a homotopy equivalence. By Proposition [3.1, the fiberwise symplectic class a extends to $M_{\mathcal{G}_{p}} \subset M_{\mathcal{G}}$. We shall denote by $\widetilde{a}_{p}$ the extension that is normalized by the requirement that $\sigma^{*}\left(\widetilde{a}_{p}\right)=0$. Correspondingly there are characteristic classes

$$
\nu_{k}:=\pi_{!}\left(\left(\widetilde{a}_{p}\right)^{k+n}\right) \in H^{2 k}\left(B \operatorname{Ham}(M, a)_{p}\right), \quad k \geq 1 .
$$

We show at the end of Section 3.2 that $\nu_{1}$ need not vanish.

\subsection{Calculations for circle actions}

We suppose that $S^{1}$ acts smoothly on a connected almost symplectic manifold $(M, a)$. By averaging we may construct an $S^{1}$-invariant closed representative $\omega$ of the class $a$. If $\underline{\xi}$ is the generating vector field on $M$ for the action then the identity

$$
0=\mathcal{L}_{\underline{\xi}}(\omega)=d\left(\iota_{\underline{\xi}} \omega\right)+\iota_{\underline{\xi}}(d \omega)
$$

implies that the 1 -form $\iota_{\xi} \omega$ is closed. To say the action is c-Hamiltonian is equivalent to saying that this 1 -form is exact. The requirements

$$
d H=\iota_{\underline{\xi}} \omega, \quad \int_{M} H \omega^{n}=0
$$

define a unique function $H: M \rightarrow \mathbb{R}$ that is called the normalized (Hamiltonian) $\omega$-moment map. The following useful result is presumably well known.

Lemma 3.5 If the action is nontrivial (ie nonconstant) $H$ cannot be identically zero.

Proof There is a subset $M_{0}$ of full measure in $M$ where the $S^{1}$ action gives rise to a fibration $S^{1} \rightarrow M_{0} \rightarrow N$. If $\omega$ is both invariant and such that $\iota_{\underline{\xi}} \omega \equiv 0$ then $\left.\omega\right|_{M_{0}}$ pulls back from $N$ and so cannot satisfy the condition $\int_{M} \omega^{\underline{n}} \neq 0$.

We first describe a cocycle representing the coupling class in the Cartan model of the equivariant cohomology (see [12] for details). Recall that the Cartan model is the following DGA

$$
\Omega_{S^{1}}^{*}(M):=\left(S\left(\operatorname{Lie}\left(S^{1}\right)^{*}\right) \otimes \Omega^{*}(M)\right) S^{1},
$$

with differential $d:=1 \otimes d_{M}-x \otimes \iota_{\underline{\xi}}$, where $\xi \in \operatorname{Lie}\left(S^{1}\right)$ is a basis vector, $x \in S\left(\operatorname{Lie}\left(S^{1}\right)^{*}\right)$ its dual and $\underline{\xi}$ is the induced vector field on $M$. The coupling 
class is represented by $\Omega:=1 \otimes \omega-x \otimes H$, where $H: M \rightarrow \mathbb{R}$ is the normalized (ie $\left.\int_{M} H \omega^{n}=0\right) \omega$-moment map. Note that, by definition of the moment map,

$$
d \Omega=-x \otimes \iota_{\underline{\xi}} \omega-x \otimes d_{M} H=0 .
$$

Thus $\Omega$ is closed.

The fiber integration $\pi_{!}: \Omega_{S^{1}}^{*}(M) \rightarrow \Omega_{S^{1}}^{*}(p t)=S\left(\operatorname{Lie}\left(S^{1}\right)^{*}\right)$ corresponds to the (equivariant) constant map $M \rightarrow p t$ in the following way. An equivariant differential form $\alpha \in \Omega_{S^{1}}^{*}(M)$ can be regarded as a polynomial map $\alpha$ : $\operatorname{Lie}\left(S^{1}\right) \rightarrow$ $\Omega^{*}(M)$. Then $\pi_{!}(\alpha)$ is a polynomial map $\operatorname{Lie}\left(S^{1}\right) \rightarrow \mathbb{R}$ given by

$$
\left(\pi_{!}(\alpha)\right)(\xi):=\int_{M} \alpha(\xi)
$$

since the (nonequivariant) fiber integration corresponding to the constant map is just the usual integration over the manifold $\int_{M}: \Omega^{*}(M) \rightarrow \Omega^{*}(p t)=\mathbb{R}$ (cf. Theorem 10.1.1 in [12]).

Lemma 3.6 Suppose that $(M, a)$ is a c-symplectic manifold of dimension $2 n$ and consider a c-Hamiltonian circle action $\lambda$ on $M$ with normalized $\omega$-moment map $H$. Denote by $\mu_{k}(\lambda) \in H_{S^{1}}^{*}(p t)$ the pullback of the characteristic class $\mu_{k}$ to $B S^{1}$ by the classifying map of the associated bundle $M \rightarrow P \rightarrow B S^{1}$. Then

$$
\mu_{k}(\lambda)=(-1)^{k}\left(\begin{array}{c}
n+k \\
n
\end{array}\right) \int_{M} H^{k} \omega^{n} \cdot x^{k} \in S\left(\operatorname{Lie}\left(S^{1}\right)^{*}\right)=H_{S^{1}}^{*}(p t) .
$$

In particular, $\mu_{k}(\lambda) \neq 0$ for even $k$ whenever the circle action is nontrivial.

Proof Because $\omega^{m}=0$ for $m>n$ for dimensional reasons,

$$
\begin{aligned}
\Omega^{n+k} & =(1 \otimes \omega-x \otimes H)^{n+k} \\
& =\sum_{m=0}^{n}(-1)^{n+k-m}\left(\begin{array}{c}
n+k \\
m
\end{array}\right)\left(x^{n+k-m} \otimes \omega^{m} H^{n+k-m}\right) .
\end{aligned}
$$

Now apply the above discussion to see that the only term that contributes to the integral is the one containing the $n$-th power of the symplectic form. This establishes formula (3.3). Now observe that the function $H^{k}$ is nonnegative for even $k$ and so by Lemma 3.5its integral vanishes only if the action is trivial.

We now complete the proof of Theorem 1.1.

Corollary 3.7 Let $\mathcal{H}$ be as in Definition 3.3 and suppose that $\lambda \subset \mathcal{H}$ is an inessential (nontrivial) circle action on $M$. Denote by $\bar{\rho} \in \pi_{4}(B \mathcal{H})$ the element $\{\Lambda, \Lambda\}$ that is formed from the suspension $\Lambda: S^{2} \rightarrow B \mathcal{H}$ of $\lambda$ as in Proposition 2.1. Then

$$
\mu_{2}(\bar{\rho}) \neq 0
$$


Proof Denote by $r: S^{1} \rightarrow \mathcal{H}$ the homomorphism with image $\lambda$. As remarked after Corollary 2.4 it suffices to show that the corresponding map $R: B S^{1} \rightarrow$ $B \mathcal{H}$ is nontrivial on $H_{4}$. But it follows from Lemma 3.6 that the pullback $R^{*}\left(\mu_{2}\right)=\left[\pi_{!}\left(\Omega^{n+2}\right)\right]$ is nonzero, where $\mu_{2}$ is as in (3.1).

The next result is an immediate consequence of Lemma 3.6 and the proof of Lemma 2.5

Lemma 3.8 Suppose that $G:=S U(\ell)$ acts smoothly on the $2 n$-manifold $(M, a)$, and let $\mathcal{H}$ be as in Definition [3.3. Denote by $\lambda_{k}, k=1, \ldots, \ell-1$, the circles in $S U(\ell)$ defined by (2.3) and by $H_{k}, k=1, \ldots, \ell-1$, the $\omega$-moment maps for $\lambda_{k}$ where $\omega$ is a $G$-invariant representative for $a$. Then $\pi_{2 k}(B G)$ has nonzero image in $H_{2 k}(B \mathcal{H})$ if and only if

$$
\int_{M}\left(H_{1}\right)^{2} H_{2} \cdots H_{k-1} \omega^{n} \neq 0 .
$$

Proof of Proposition 1.4 This is the special case with $\ell=2$. Then the integral is $\int H_{1}^{2} \omega^{n}$ which is always nonzero by Lemma 3.5

Proof of Lemma 1.3 Consider a c-Hamiltonian $S^{1}$-action with moment map $H$, and choose a fixed point $p$. Because $S^{1}$ maps to a loop in Diff ${ }_{0}$ with trivial flux the inclusion $S^{1} \rightarrow$ Diff $_{0}$ lifts to a homomorphism $S^{1} \rightarrow \mathcal{G}_{p}$, where $\mathcal{G}_{p}$ is as defined in Remark [3.4. Moreover, the section $\sigma$ pulls back over the corresponding classifying map $B S^{1} \rightarrow B \mathcal{G}_{p}$ to the section of $M_{S^{1}} \rightarrow B S^{1}$ with image $B S^{1} \times\{p\}$. Therefore the pullback of $\widetilde{a}_{p}$ to $M_{S^{1}}$ may be represented in the Cartan model by the element $\Omega_{a}:=1 \otimes \omega-x \otimes(H-H(p))$. Hence the proof of Lemma 3.6 shows that for some nonzero constant $c$

$$
\nu_{1}(\lambda)=c . \int_{M}(H-H(p)) \omega^{n} .
$$

If this integral is nonzero, the 2 -skeleton $S^{2}$ of $B S^{1}$ maps nontrivially to $B \mathcal{G}_{p}$ under the classifying map $B S^{1} \rightarrow B \mathcal{G}_{p}$. This is equivalent to saying that $\lambda$ is essential in $\mathcal{G}_{p}$.

\subsection{Relation to Reznikov's classes and the $\kappa$-classes}

Above we defined classes $\mu_{k}$ in $H^{*}(B \mathcal{H})$ for monoids $\mathcal{H}$ whose action is cHamiltonian, ie, the c-symplectic class $a$ extends over $M_{\mathcal{H}}$. If we restrict to the case $\mathcal{G}=\operatorname{Ham}(M, \omega)$ then these classes have a geometric interpretation. 
We now show that they can be constructed by the usual Chern-Weil process. As a consequence they desuspend to classes in $H^{2 k-1}(\operatorname{Ham}(M, \omega)$ ) (the usual singular cohomology of $\operatorname{Ham}(M, \omega)$ considered as a topological space) that are the restrictions of Reznikov's classes in the smooth Lie algebra cohomology $H_{s m}^{2 k-1}(\operatorname{Lie} \operatorname{Ham}(M, \omega))$.

To understand this, recall that $\operatorname{Lie} \operatorname{Ham}(M, \omega)$ can be identified with the space of functions $C_{0}(M)$ of zero mean on $M$ (with respect to the volume form $\omega^{n}$.) Moreover, it has a nondegenerate bilinear form (a Killing form) defined by

$$
\langle F, G\rangle:=\int_{M} F G \omega^{n}
$$

that is invariant under the adjoint action of $\mathcal{G}:=\operatorname{Ham}(M, \omega)$. Now the coupling class $[\Omega]$ may be represented by a closed differential form $\Omega$ on $M_{\mathcal{G}}$ that extends the fiberwise symplectic form. (Here we are working with de Rham theory on $M_{\mathcal{G}}$ which is not, strictly speaking, a manifold. However, one can make everything precise by considering an arbitrary smooth map $B \rightarrow B \mathcal{G}$ and looking at the associated smooth pullback bundle over $B$.) The form $\Omega$ defines a connection on the bundle $\pi: M_{\mathcal{G}} \rightarrow B_{\mathcal{G}}$ whose horizontal distribution $H_{o r}, x \in M_{\mathcal{G}}$, is given by the $\Omega$-orthogonals to the vertical tangent bundle. As shown by Guillemin-Lerman-Sternberg [11, the corresponding parallel transport maps preserve the fiberwise symplectic form and the holonomy is Hamiltonian. Moreover, given vector fields $v, w \in T_{b} B \mathcal{G}$ with horizontal lifts $v^{\sharp}, w^{\sharp}$, the function $\Omega\left(v^{\sharp}, w^{\sharp}\right)(x)$ restricts on each fiber $M_{b}:=\pi^{-1}(b)$ to an element of $\operatorname{Lie}(\mathcal{G})$ that represents the curvature of this connection at $(v, w)$. In other words, the closed 2 -form $\Omega$ on $M_{\mathcal{G}}$ defines a 2 -form $\widetilde{\Omega}$ on the base $B \mathcal{G}$ of the fibration that takes values in the Lie algebra $\operatorname{Lie}(\mathcal{G})$ of the structural group $\mathcal{G}$ and is a curvature form in the usual sense. (For more detail, see McDuff-Salamon [18, Ch 6].)

Any Ad-invariant polynomial $\mathcal{I}^{k}: \operatorname{Lie}(\mathcal{G})^{\otimes k} \rightarrow \mathbb{R}$ therefore gives rise to a characteristic class $c_{k}^{\mathcal{G}}$ in $H^{*}(B \mathcal{G})$, namely the class represented by the closed realvalued $2 k$-form $\mathcal{I}^{k} \circ \widetilde{\Omega}^{k}$. In the case at hand we may take

$$
\mathcal{I}^{k}\left(F_{1} \otimes \cdots \otimes F_{k}\right):=\int_{M} F_{1} \cdots F_{k} \omega^{n}
$$

Lemma 3.9 This class $c_{k}^{\mathcal{G}}$ equals const. $\mu_{k}$.

Proof Let $v_{1}, \ldots, v_{2 k}$ be vector fields on $B \mathcal{G}$ with horizontal lifts $v_{1}^{\sharp}, \ldots, v_{2 k}^{\sharp}$. 
Then, if the $w_{j}$ are tangent to the fiber at $x \in M_{\mathcal{G}}$ we find

$$
\begin{aligned}
\Omega^{n+k}\left(w_{1}, \ldots, w_{2 n}, v_{1}^{\sharp}, \ldots, v_{2 k}^{\sharp}\right)(x)= & \sum_{\sigma} \varepsilon(\sigma)\left(\begin{array}{c}
n+k \\
n
\end{array}\right) \times \\
& F_{1, \sigma}(x) \cdots F_{k, \sigma}(x) \omega^{n}\left(w_{1}, \ldots, w_{2 n}\right),
\end{aligned}
$$

where, for each permutation $\sigma$ of $\{1, \ldots, 2 k\}, \varepsilon(\sigma)$ denotes its signature and

$$
F_{j, \sigma}(x):=\Omega\left(v_{\sigma(2 j-1)}^{\sharp}, v_{\sigma(2 j)}^{\sharp}\right)(x)=\widetilde{\Omega}\left(v_{\sigma(2 j-1)}, v_{\sigma(2 j)}\right)(x) .
$$

Therefore $\left(\pi ! \Omega^{n+k}\right)\left(v_{1}, \ldots, v_{2 k}\right)=$ const. $\mathcal{I}^{k} \circ \widetilde{\Omega}^{k}\left(v_{1}, \ldots, v_{2 k}\right)$ as claimed.

Now suppose given a homomorphism from a Lie group $G$ to $\operatorname{Ham}(M, \omega)$. The classes $\mu_{k} \in H^{*}(B \operatorname{Ham}(M, \omega))$ pull back under the map $R: B G \rightarrow$ $B \operatorname{Ham}(M, \omega)$ to some elements in $H^{*}(B G)$, which by definition are $G$-characteristic classes. One can figure out which classes they are by investigating the invariant polynomial

$$
\operatorname{Lie}(G)^{\otimes k} \longrightarrow C_{0}(M)^{\otimes k} \stackrel{\mathcal{I}^{k}}{\longrightarrow} \mathbb{R} .
$$

Reznikov 22] did this calculation (in a slightly different context) for the case of the action of $S U(n+1)$ on $\mathbb{C P}^{n}$, and concluded that the $\mu_{k}$ pull back to algebraically independent elements in $H^{*}(B S U(n+1))$. The advantage of our approach is that the classes extend to $B \widetilde{\mathcal{H}}_{0}^{a}$ (and when $H^{1}(M)=0$ to $B \mathcal{H}_{a}$ ) since one does not use the action of the Lie algebra in their definition.

There are other characteristic classes in $H^{*}(B \mathcal{G})$ arising from the characteristic classes on $M$ preserved by the elements of $\mathcal{G}$, ie the Chern classes when $\mathcal{G}=$ $\operatorname{Symp}(M, \omega)$ or $\operatorname{Ham}(M, \omega)$, the Pontriagin classes when $\mathcal{G}=\operatorname{Diff}(M)$, and the Euler class in the case of $\widetilde{\mathcal{H}}_{0}^{a}$. For example, in the Hamiltonian (but not the c-Hamiltonian) case we can use the Chern classes of the tangent bundle $T M$. Each Chern class $c_{i}(M)$ has a natural extension $\widetilde{c}_{i}$ to $M_{\mathcal{G}}$, namely the $i$ th Chern class of the vertical tangent bundle. Hence for each multi-index $I:=\left(m_{1}, \ldots, m_{n}\right)$ there is a class

$$
\mu_{k, I}:=\pi_{!}\left(\Omega^{k}\left(\widetilde{c}_{1}\right)^{m_{1}} \ldots\left(\widetilde{c}_{n}\right)^{m_{n}}\right) \in H^{*}(B \mathcal{G}) .
$$

As indicated above, initially these classes live on the connected group $\mathcal{G}:=$ $\operatorname{Ham}(M, \omega)$. If $H^{1}(M)=0$ they extend to the full symplectomorphism group $\operatorname{Symp}(M)$, but in general only the classes with $k=0$ extend over this group. If $M$ is a Riemann surface $\Sigma$ and $I=m$ then the classes $\kappa_{m}:=\mu_{0, m+1}$ are known as the Miller-Morita-Mumford $\kappa$-classes. If $\Sigma$ has genus $g>1$ with orientation class $a$ normalized by $\int_{\Sigma} a=1$, then $\operatorname{Symp}(M)$ and $\mathcal{H}_{a}$ are homotopy 
equivalent to the (orientation preserving) mapping class group $\pi_{0}(\operatorname{Symp}(\Sigma))$. Further

$$
\widetilde{a}=\frac{1}{2-2 g}\left(\widetilde{c}_{1}+\frac{1}{4 g-4} \pi^{*}\left(\kappa_{1}\right)\right) .
$$

Hence the $\mu_{k}, k>1$, together with the single class $\kappa_{1}$ contain the same information as the $\kappa$-classes.

\section{Higher homotopy groups}

We begin with examples of nontrivial $G=S U(\ell)$ actions for which the map $\pi_{*}(G) \rightarrow \pi_{*}(\mathcal{G})$ is not injective, and then discuss an easy way to detect elements in $\pi_{*}(\mathcal{G})$. Section 4.2 proves Proposition 1.5 and Section 4.3 concerns flag manifolds.

\subsection{Examples}

Example 4.1 (Action of $G=S U(\ell)$ with $r_{*}=0$ in dimensions $1 \bmod 4$ ) Let $2 \ell \leq n+1$ and consider the action of $S U(\ell)$ on $\mathbb{C P}^{n}$ given by restricting the standard action of $S U(n+1)$ to the image of the homomorphism

$$
\rho: G=S U(\ell) \rightarrow S U(n+1): A \mapsto(A, \bar{A}) \in S U(\ell) \times S U(\ell) \subset S U(n+1),
$$

where $\bar{A}$ denotes the conjugate of $A$. Since conjugation is an automorphism of $S U(\ell)$ that acts by -1 on the homotopy groups in degrees $\equiv 1(\bmod 4)$, the induced map $\rho_{*}: \pi_{j}(G) \rightarrow \pi_{j}(S U(n+1))$ is zero when $j \equiv 1(\bmod 4)$. Hence the image of $\pi_{j}(G)$ in $\pi_{j}\left(\operatorname{Ham}\left(\mathbb{C P}^{n}\right)\right)$ vanishes for these $j$. Note that the pullback of the universal bundle $E_{n+1} \rightarrow B U(n+1)$ by $B \rho: B U(\ell) \rightarrow$ $B U(n+1)$ is the sum $E_{\ell} \oplus E_{\ell}^{*} \oplus \mathbb{C}^{n+1-2 \ell}$. Thus this example reformulates the fact that the odd Chern classes of a bundle of the form $E_{\mathbb{R}} \otimes \mathbb{C}$ vanish (rationally), where $E_{\mathbb{R}}$ denotes the real bundle underlying $E$.

One can also find representations $\rho: U(\ell) \rightarrow U(n+1)$ that kill homotopy in dimensions $4 i+3$, and hence actions of $U(\ell)$ on projective space with the same property.

Example 4.2 (Action of $S U(4)$ with $r_{*}=0$ in dimension 7) If $E \rightarrow$ $B U(4)$ is the universal bundle and $\left.\gamma: S^{8} \rightarrow B U(4)\right)$ generates $\pi_{8}(B U(4))$, then $c_{4}(E)(\gamma)=6$ by definition. We claim that

$$
c_{4}\left(\Lambda^{2}(E)\right)(\gamma)=-24
$$


To check this, pull $c_{4}\left(\Lambda^{2}(E)\right)$ back to $H^{*}(B \mathbb{T})$ where $\mathbb{T}$ is the maximal 4torus in $U(4)$. If $t_{1}, \ldots, t_{4}$ denote the obvious generators of $H^{2}(B \mathbb{T})$ then the pullback of $c_{4}\left(\Lambda^{2}(E)\right)$ to $\left.H^{8}(B \mathbb{T})\right)$ is

$$
2 s_{3,1}+5 s_{2,2}+13 s_{2,1,1}+30 s_{1,1,1,1}
$$

where, for each partition $I=\left(i_{1}, \ldots, i_{r}\right)$ of the number $4, s_{I}$ denotes the symmetric function $\sum t_{1}^{i_{1}} \cdots t_{r}^{i_{r}}$. When we express this in terms of the elementary symmetric functions $\sigma_{i}$ and pull back by $\gamma$, the only contribution comes from the terms in $\sigma_{4}$. Thus

$$
c_{4}\left(\Lambda^{2}(E)\right)(\gamma)=6(2 \cdot 4+5 \cdot 2+13 \cdot(-4)+30)=-24 .
$$

Therefore the homomorphism $U(4) \rightarrow U(22)$ that classifies the sum of four copies of the rank 4 bundle $E$ with the rank 6 bundle $\Lambda^{2}(E)$ kills $\pi_{7}(U(4))$. A similar statement holds for the corresponding action of $S U(4)$ on $\mathbb{C P}^{21}$.

The next result gives a way of detecting the image in $\pi_{*}(\mathcal{G})$ of some of the elements that come from $\pi_{*}\left(G_{p}\right)$. Consider the commutative diagram

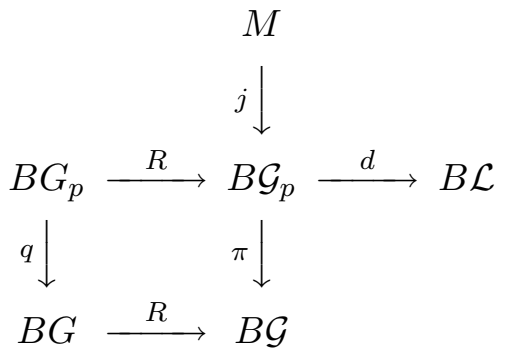

where $\mathcal{G}$ denotes either $\operatorname{Ham}(M, \omega)$ or $\operatorname{Diff}_{0}(M)$ as appropriate, and $\mathcal{L}$ is the group formed by the linearized action of $\mathcal{G}_{p}$ on $T_{p} M$. Thus when $\mathcal{G}$ consists of symplectomorphisms $\mathcal{L}=S p(2 n) \simeq U(n)$, while in the smooth case $\mathcal{L}=$ $G L(2 n, \mathbb{R}) \simeq S O(2 n)$. The homomorphism $d: \mathcal{G}_{p} \rightarrow \mathcal{L}$ is given by taking the derivative, so that the composite $d \circ j: M \rightarrow B \mathcal{G}_{p} \rightarrow B \mathcal{L}$ classifies the tangent bundle of $M$. In degrees in which this vanishes in homotopy we can use the map $d \circ R$ to detect the image of $R_{*}$. For clarity, we state the next lemma in the symplectic case. Thus $\mathcal{G}:=\operatorname{Ham}(M, \omega)$ and $\mathcal{L}:=U(n)$ where $2 n=\operatorname{dim} M$.

Lemma 4.3 Suppose that the Lie group $G:=S U(\ell)$ acts on $M$, and consider the associated map $r: G \rightarrow \mathcal{G}$. Assume that $(d \circ j)_{*}: \pi_{k}(M) \rightarrow \pi_{k}(B \mathcal{L})$ vanishes, and that there is $\bar{\alpha} \in \pi_{k}\left(B G_{p}\right)$ such that both $q_{*}(\bar{\alpha}) \in \pi_{k}(B G)$ and $(d \circ R)_{*}(\bar{\alpha}) \in \pi_{k}(B \mathcal{L})$ are nonzero. Then $R_{*}: \pi_{k}(B G) \otimes \mathbb{Q} \rightarrow \pi_{k}(B \mathcal{G}) \otimes \mathbb{Q}$ is injective. 
Proof The proof is an easy diagram chase. It suffices to show that

$$
(R \circ q)_{*}(\bar{\alpha})=(\pi \circ R)_{*}(\bar{\alpha})
$$

is nonzero. But otherwise $R_{*}(\bar{\alpha})$ would be in the kernel of $\pi_{*}$ and hence in the image of $j_{*}$. This would imply $d_{*}\left(R_{*}(\bar{\alpha})\right)=d_{*}\left(j_{*}(\beta)\right)=0$, which contradicts the assumption that $d_{*}\left(R_{*}(\bar{\alpha})\right) \neq 0$.

We apply this to flag manifolds in Lemma 4.7 below.

\section{2 c-split manifolds and the evaluation map}

We now prove Proposition 1.5 that detects the image of elements in $\pi_{*}(G)$ that map nontrivially under the evaluation map. Recall from Lalonde-McDuff [14] that a fibration $M \rightarrow P \rightarrow B$ is said to be c-split if $\pi_{1}(B)$ acts trivially on $H^{*}(M)$ and the Leray-Serre spectral sequence degenerates at the $E_{2}$ term. This is equivalent to the condition that the inclusion induces an injective map $H_{*}(M) \rightarrow H_{*}(P)$. Further a c-symplectic manifold $(M, a)$ is said to satisfy the hard Lefschetz condition if

$$
a^{k} \cup: H^{n-k}(M) \rightarrow H^{n+k}(M)
$$

is an isomorphism for all $1 \leq k \leq n$.

Lemma 4.4 Suppose that $(M, a)$ satisfies the hard Lefschetz condition and denote by $\mathcal{H}$ either $\widetilde{\mathcal{H}}_{0}^{a}$ or (if $H^{1}(M)=0$ ) the monoid $\mathcal{H}_{H}$ of all homotopy equivalences that act trivially on rational homology. Then $M \rightarrow M_{\mathcal{H}} \rightarrow B \mathcal{H}$ is c-split.

Proof It is a classical result due to Blanchard 6 that every fibration over a base $B$ such that $\pi_{1}(B)$ acts trivially on the homology of the fiber is c-split provided that the class $a$ extends to $P$ and the fiber $(M, a)$ satisfies the hard Lefschetz condition. Hence we need only check that a extends to $M_{\mathcal{H}}$, which is true by Proposition 3.1 .

Proof of Proposition 1.5 Consider the fibration $M \rightarrow M_{\mathcal{H}} \rightarrow B \mathcal{H}$. The boundary map $\partial: \pi_{*}(B \mathcal{H}) \rightarrow \pi_{*-1}(M)$ of its long exact homotopy sequence is essentially the same as the evaluation map ev $: \pi_{*}(\mathcal{H}) \otimes \mathbb{Q} \rightarrow \pi_{*}(M) \otimes \mathbb{Q}$ : more precisely, ev $=\partial \circ \tau$ where $\tau: \alpha \rightarrow \bar{\alpha}$ is given by suspension. Therefore, we must show that if a connected group $G$ acts on a nilpotent manifold $(M, a)$ that satisfies the c-splitting condition for $\mathcal{H}$, then for every element 
$\alpha \in \pi_{2 k-1}(G), k>1$, with $\operatorname{ev}_{*}(\alpha) \neq 0$ the element $h(\bar{\alpha}) \in H_{*}(B \mathcal{H})$ is nonzero. To this end, let $P \rightarrow S^{2 k}$ be the pullback of $M_{\mathcal{H}} \rightarrow B \mathcal{H}$ by $\bar{\alpha}$, and consider the commutative diagram:

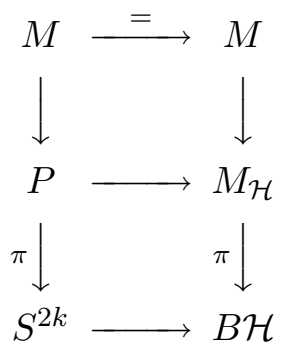

By hypothesis the fibration $M \rightarrow M_{\mathcal{H}} \rightarrow B \mathcal{H}$ is c-split. Hence the elements of any additive basis $b_{0}:=1, b_{1}, \ldots, b_{q}$ for $H^{*}(M)$ extend to elements $\widetilde{b}_{0}:=$ $1, \ldots, \widetilde{b}_{q}$ of $H^{*}\left(M_{\mathcal{H}}\right)$. Moreover, if $\operatorname{deg} b_{i}<2 k$ the restriction of $\widetilde{b}_{i}$ to $P$, which we denote by $\widetilde{b}_{i}^{P}$, is uniquely determined.

Let $e \in H^{2 k}\left(S^{2 k}\right)$ be a generator. Then $\pi^{*}(e) \neq 0$ since the fibration $P \rightarrow S^{2 k}$ c-splits. We claim that there is a polynomial $f$ such that the following relation holds in $H^{*}(P)$ for suitable $i_{1}, \ldots, i_{s}$ :

$$
\pi^{*}(e)=f\left(\widetilde{b}_{i_{1}}^{P}, \ldots, \widetilde{b}_{i_{s}}^{P}\right), \quad \operatorname{deg} b_{i_{j}}^{P}<2 k, \quad \forall j .
$$

To see this, build the KS model $\left(\mathcal{A}_{P}, D\right)$ for the fibration $M \rightarrow P \rightarrow S^{2 k}$. According to Tralle-Oprea 24, this has the form $\left(\Lambda(e, t) \otimes \mathcal{A}_{M}, D\right)$ where $\left(\mathcal{A}_{M}, d_{M}\right)$ is a minimal model for $H^{*}(M),(\Lambda(e, t), d)$ is a minimal model for $S^{2 k}$ (so that $\operatorname{deg} e=2 k, \operatorname{deg} t=4 k-1$ ), and $D$ extends $d_{M}$. Since $M$ and hence $P$ is nilpotent, this KS model calculates $H^{*}(P)$. Since it has generators in dimensions $\geq 2$ that are dual to the rational homotopy, it agrees with $\mathcal{A}_{M}$ in dimensions $<2 k-1$. In dimension $2 k-1$ we may choose generating cochains for $\mathcal{A}_{M}^{2 k-1}$ so that precisely one of them, say $x$, does not vanish on the image of $\partial: \pi_{2 k}\left(S^{2 k}\right) \rightarrow \pi_{2 k-1}(M)$. Then $D(x)=e+r$ where $r=d_{M}(x)$ is a product of elements in $\mathcal{A}_{M}$ of degrees $<2 k$. Since $0=D^{2}(x)=D(e)+D(r)=D(r), r$ is a cocycle in $\mathcal{A}_{P}$. Moreover, because $\pi^{*}(e) \neq 0, r$ must represent a nontrivial class in $H^{*}(P)$. Therefore if $f$ is the polynomial such that $f\left(b_{i_{1}}, \ldots, b_{i_{s}}\right)=-r$, relation (4.1) holds.

Now consider the corresponding element $f\left(\widetilde{b}_{i_{1}}, \ldots, \widetilde{b}_{i_{s}}\right) \in H^{*}\left(M_{\mathcal{H}}\right)$. Since $\pi: M_{\mathcal{H}} \rightarrow B \mathcal{H}$ is c-split, we may apply the Leray-Hirsch theorem. Therefore this can be written uniquely in the form

$$
f\left(\widetilde{b}_{i_{1}}, \ldots, \widetilde{b}_{i_{s}}\right)=\sum_{j=0}^{q} \pi^{*}\left(z_{j}\right) \widetilde{b}_{j},
$$


where $z_{j} \in H^{*}(B \mathcal{H})$. If we pull this relation back to $P$ we obtain the unique expression for $f\left(\widetilde{b}_{i_{1}}^{P}, \ldots, \widetilde{b}_{i_{s}}^{P}\right)$ in terms of the Leray-Hirsch basis $\widetilde{b}_{j}^{P}$ for $H^{*}(P)$. Comparing with (4.1), we find that $z_{0} \in H^{2 k}(B \mathcal{H})$ must extend $e$. Thus $\bar{\alpha}$ has nonzero image in $H_{*}(B \mathcal{H})$.

Remark 4.5 (i) Equation (4.1) implies that $\pi_{!}\left(f\left(\widetilde{b}_{i_{1}}^{P}, \ldots, \widetilde{b}_{i_{s}}^{P}\right)\right)=e$. In fact the fiber integral simply picks out the coefficient of $b_{0}:=1$ in the Leray-Hirsch decomposition for an element in $H^{*}\left(M_{\mathcal{H}}\right)$.

(ii) The c-splitting hypothesis in Proposition 1.5 is satisfied when $M$ is a c-symplectic manifold with a transitive action of a connected and simply connected compact Lie group $G$. For if $\omega$ is a $G$-invariant representative for the c-symplectic class $a \in H^{2}(M)$, then $\omega^{n}$ has constant rank and hence does not vanish. Thus $\omega$ is symplectic. Our assumptions imply that $G$ is semisimple and that $\pi_{1}(M)=0$. Therefore $M$ has a Kähler structure with Kähler form $\omega$ by a theorem of Borel that is stated for example in Tralle-Oprea [24, Ch 5,Thm 2.1]. Therefore the claim follows from Lemma 4.4.

We now give a version of Proposition 1.5 in which the c-splitting condition is relaxed. All we need is that the cohomology classes in $M$ occuring in the relation (4.11) extend to $H^{*}\left(M_{\mathcal{H}}\right)$. In particular, if in the statement below $H^{*}(X)$ is generated by the Chern classes of $M$ then we can forget about the class $[\omega]$ and take $\mathcal{G}:=\operatorname{Symp}(M)$ even if $H^{1}(M) \neq 0$.

Proposition 4.6 Suppose that a simply connected group $G$ acts on $(M, \omega)$ in such a way that the subring of $H^{*}(M)$ generated by $[\omega]$ and the Chern classes of $T M$ surjects onto $H^{*}(X)$, where $X:=G / G_{p}$ is some orbit of $G$. Let $\mathcal{G}$ be a subgroup of $\operatorname{Symp}(M)$ that contains $G$ and is such that $[\omega]$ extends to $H^{*}\left(M_{\mathcal{G}}\right)$. Then the map

$$
h \circ R_{*}: \pi_{*}(B G) \rightarrow H_{*}(B \mathcal{G})
$$

is nonzero on all elements $\bar{\alpha} \in \pi_{2 k}(B G)$ such that $\mathrm{ev}_{*}(\alpha) \in \pi_{2 k-1}(M)$ is nonzero.

Proof Suppose given $\alpha \in \pi_{2 k-1} G$ such that $\operatorname{ev}_{*}(\alpha) \neq 0$ in $\pi_{2 k-1}(M)$ and 
hence also in $\pi_{2 k-1}(X)$. Consider the corresponding diagram:

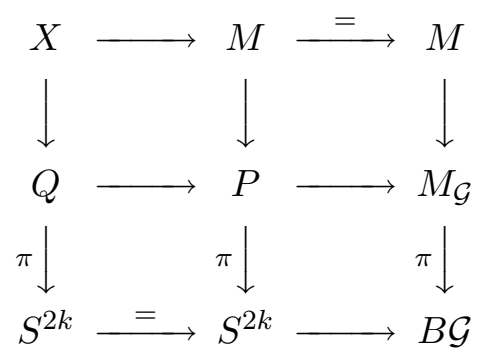

Our hypotheses ensure that there is a basis $b_{0}:=1, b_{1}, \ldots, b_{N}$ for $H^{*}(X)$ consisting of elements that extend to $H^{*}\left(M_{\mathcal{G}}\right)$. Hence the fibration $\pi: Q \rightarrow S^{2 k}$ is c-split and the result follows by arguing as in the proof of Proposition 1.5 .

\subsection{Flag manifolds}

Consider the general flag manifold

$$
M\left(m_{1}, \ldots, m_{k}\right):=U(\ell) / U\left(m_{1}\right) \times \cdots \times U\left(m_{k}\right), \quad m_{1} \geq \cdots \geq m_{k},
$$

where $\ell=\sum m_{i}$. We shall denote

$$
G^{\prime}:=U(\ell), \quad G_{p}^{\prime}:=U\left(m_{1}\right) \times \cdots \times U\left(m_{k}\right), \quad G:=S U(\ell), \quad G_{p}:=G_{p}^{\prime} \cap G .
$$

If $E_{i} \rightarrow U\left(m_{i}\right)$ denotes the universal bundle, then the vertical tangent bundle of the fibration

$$
M \stackrel{j}{\longrightarrow} B G_{p} \stackrel{q}{\longrightarrow} B G
$$

is the pullback from $B G_{p}^{\prime}$ to $B G_{p}$ of the bundle

$$
\bigoplus_{1 \leq i<j \leq k} \operatorname{Hom}\left(E_{i}, E_{j}\right)=\bigoplus_{1 \leq i<j \leq k} E_{i}^{*} \otimes E_{j}
$$

Further, the cohomology ring of $M$ is generated by the Chern classes of the $E_{i}$ with defining relations coming from the fact that the restriction of $\oplus_{i} E_{i}$ to $M$ is trivial. In particular, in the case of a Grassmannian (ie $k=2$ ) the cohomology of $M$ is generated by the Chern classes of its tangent bundle so that $\operatorname{Symp}(M, \omega)$ acts trivially on $H^{*}(M)$.

The first part of the next lemma illustrates the use of Lemma 4.3. though in this case stronger conclusions may be obtained by other methods (see Proposition 4.8.) Recall that $\mathcal{H}_{H}$ denotes the space of homotopy equivalences that act trivially on $H^{*}(M)$. 
Lemma 4.7 Let $M$ be the flag manifold $M\left(m_{1}, \ldots, m_{k}\right)$ and set $G:=S U(\ell)$ where $\ell=\sum m_{i}$ and $m_{1} \geq \cdots \geq m_{k}$. Then:

(i) $r_{*}: \pi_{2 i-1}(G) \rightarrow \pi_{2 i-1}(\operatorname{Symp}(M, \omega))$ is injective for $m_{2}<i \leq m_{1}$.

(ii) $h \circ R_{*}: \pi_{2 i}(B G) \rightarrow H_{2 i}\left(B \mathcal{H}_{H}\right)$ is injective for $i=2$ and $m_{1}<i \leq \ell$.

Proof The existence of the fibration

$$
M \rightarrow B G_{p} \rightarrow B G
$$

implies that the generators of $\pi_{*}(M)$ divide into two groups. Those in dimensions $2 i, 1 \leq i \leq m_{2}$, map to the elements in the kernel of $\pi_{*}\left(B G_{p}\right) \rightarrow \pi_{*}(B G)$, while the generators in the image of $\mathrm{ev}_{*}$ come from $\pi_{*+1}(B G)$ and lie in odd dimensions $2 i-1$ for $m_{1}<i \leq \ell$. Therefore because $\pi_{2 i}(M)=0$ in the range $m_{2}<i \leq m_{1}$, the first statement will follow from Lemma 4.3 if we show that $(d \circ r)_{*}: \pi_{2 i}\left(B G_{p}\right) \rightarrow \pi_{2 i}(B \mathcal{L})$ is surjective for $m_{2}<i \leq m_{1}$. To prove this it suffices to check that the Chern classes of the vertical tangent bundle of the fibration $M \rightarrow B G_{p} \rightarrow B G$ are nonzero on $\pi_{2 i}\left(B G_{p}\right)$ for $i$ in this range. This holds because the restriction of this bundle to $B S U\left(m_{1}\right)$ is simply a sum of copies of $E_{1}^{*}$. This proves (i).

Since $\mathrm{ev}_{*}: \pi_{*}(G) \rightarrow \pi_{*}(M)$ is injective in dimensions $*>2 m_{1}-1$, (ii) follows immediately from Propositions 1.4 and [1.5] and Lemma 4.4

We next show that the map $h \circ R_{*}: \pi_{*}(B G) \rightarrow H_{*}\left(B \mathcal{H}_{H}\right)$ is always injective for flag manifolds. Our argument imitates the proof for $\mathbb{C P}^{n}$, considering the coefficients of the higher degree elements $\widetilde{b}_{j}, j>0$, in (4.2).

Proposition 4.8 Let $M$ be the flag manifold $M\left(m_{1}, \ldots, m_{k}\right)$ and set $G:=$ $S U(\ell)$ where $\ell=\sum m_{i}$ and $m_{1} \geq \cdots \geq m_{k}$. Then the inclusion $B S U(\ell) \rightarrow$ $B \mathcal{H}_{H}$ induces an injection on $H_{*}$.

Proof Let us first consider the case of a Grassmannian $M(m, k)$ where $\ell=$ $m+k, m \geq k$, and set $G:=S U(\ell)$. It is convenient to work with cohomology. Therefore we aim to show that the Chern classes $c_{i}, i=2, \ldots, \ell$ in $H^{*}(B G)$ extend to classes in $H^{*}\left(B \mathcal{H}_{H}\right)$.

As remarked above, the ring $H^{*}(B(U(m) \times U(k)))$ is freely generated by elements $x_{1}, \ldots, x_{m}$ (the pullbacks of the Chern classes of the universal bundle $\left.E:=E_{1} \rightarrow B U(m)\right)$ and $y_{1}, \ldots, y_{k}$ (the pullbacks of the Chern classes of the universal bundle $\left.F:=E_{2} \rightarrow B U(k)\right)$. Thus $H^{*}\left(B G_{p}\right)=H^{*}(B S(U(m) \times$ $U(k)))$ is the quotient of this free ring by the relation $x_{1}+y_{1}=0$. 
Consider the fibration $\pi: B G_{p} \rightarrow B G$. Since $E \oplus F$ is the pullback of the universal bundle over $B G:=B S U(\ell)$ its Chern classes are the pullbacks $Q_{i}:=$ $\pi^{*}\left(c_{i}\right)$ of the Chern classes in $H^{*}(B G)$. Thus $Q_{0}:=1$ and $Q_{1}=0$. Taking the total Chern class of $E \oplus F$ we find:

$$
\left(1+x_{1}+\cdots+x_{m}\right)\left(1+y_{1}+\cdots+y_{k}\right)=1+Q_{1}+\cdots+Q_{m+k}
$$

Since the restriction of $E \oplus F$ to $M$ is trivial, the above identity gives $m+$ $k$ relations among the restrictions of the $x_{i}, y_{j}$ to $H^{*}(M)$. The first $m$ of these should be interpreted as defining the $x_{i}$ 's in terms of the generators $y_{i}$ of $H^{*}(M)$, while the equations $Q_{m+i}=0$ give the relations in $H^{*}(M)$. In particular, there are no relations among the monomials in the $y_{i}$ in degrees $\leq 2 \mathrm{~m}$. Hence we may choose an additive basis $b_{0}:=1, b_{1}, \ldots, b_{N}$ for $H^{*}(M)$ whose elements in degrees $\leq 2 m$ consist of all the monomials in the $y_{i}$. In these degrees we may therefore write $b_{\nu}=y_{I_{\nu}}$, and will extend $b_{\nu} \in H^{*}(M)$ to $H^{*}\left(M_{G}\right)$ by identifying it with $y_{I_{\nu}} \in H^{*}\left(M_{G}\right)$. As in Proposition 1.5, the $b_{\nu}$ also extend to elements $\widetilde{b}_{\nu} \in H^{*}\left(M_{\mathcal{H}}\right)$. We denote by $\widetilde{b}_{\nu}^{G}$ the restriction of $\widetilde{b}_{\nu}$ to $M_{G}=B G_{p}$. These two extensions $\widetilde{b}_{\nu}^{G}$ and $b_{\nu}$ of $b_{\nu} \in H^{*}(M)$ to $H^{*}\left(M_{G}\right)$ need not agree, but they do agree modulo the ideal $\left\langle Q_{2}, \ldots, Q_{m}\right\rangle$ generated by the elements of $\pi^{*}(B G)$. In other words, if we identify $H^{*}\left(B G_{p}\right)$ with the free algebra generated by the $y_{j}$ and $Q_{i}, 2 \leq i \leq m$, we have

$$
b_{\nu} \in \widetilde{b}_{\nu}^{G}+\left\langle Q_{2}, \ldots, Q_{m}\right\rangle \text {. }
$$

Formally inverting $1+y_{1}+\cdots+y_{k}$ in equation (4.3) we obtain

$$
1+x_{1}+\cdots+x_{m}=\left(1+Q_{1}+\cdots+Q_{m+k}\right)\left(1+f_{1}\left(y_{1}, \ldots, y_{k}\right)+\ldots\right),
$$

where the $f_{i}$ are the homogeneous terms of degree $2 i$ in $\left(1+y_{1}+\cdots+y_{k}\right)^{-1}$. The terms of degree $2 m+2$ give the relation

$$
-Q_{m+1}=\sum_{i=1}^{m} Q_{m-i+1} f_{i}\left(y_{1}, \ldots, y_{k}\right) .
$$

Notice also that the coefficient of $y_{1}^{i}$ in $f_{i}$ equals $(-1)^{i}$. Since the polynomials $f_{i}$ have degree $2 i \leq 2 m$ they are sums $\sum \alpha_{\nu} b_{\nu}$ of the basis monomials $b_{\nu}=y_{I_{\nu}}$. Therefore we have

$$
\begin{aligned}
\pi^{*}\left(c_{m+1}\right)=Q_{m+1} & =-\sum_{i=1}^{m} Q_{m-i+1} f_{i}\left(y_{1}, \ldots, y_{k}\right) \\
& =-\sum_{i=1}^{m} Q_{m-i+1}\left(\sum_{\nu} \alpha_{\nu} b_{\nu}\right) \\
& =\sum_{\nu} \pi^{*}\left(e_{\nu}\right) \widetilde{b}_{\nu}^{G}
\end{aligned}
$$


where each $e_{\nu} \in H^{*}(B G)$ is a polynomial in the Chern classes with a nonzero linear term. Further, if $b_{\nu_{i}}$ denotes the basis element $y_{1}^{m-i}$ of $H^{*}(M)$ for $1<i \leq m$, then the coefficient of $\widetilde{b}_{\nu_{i}}^{G}$ in the above expression has the form $\mu c_{i}+$ decomposables, for some nonzero number $\mu$.

It follows from Proposition [1.5 that there is a class $u \in H^{2 m+2}(B \mathcal{H})$ whose restriction to $B G$ does not vanish on $\pi_{2 m+2}(B G)$. Hence we may choose $u$ so that it restricts to $c_{m+1}+p(c) \in H^{2 m+2}(B G)$ where $p(c)$ is some polynomial in the $c_{i}, 2 \leq i \leq m$. Thus $\pi^{*}(u)$ restricts to $Q_{m+1}+\pi^{*}(p(c))$ where $\pi^{*}(p(c))$ is a polynomial in the $Q_{i}, i \leq m$. Since $\pi^{*}(u)$ vanishes on the fiber $M$ and there is a unique relation in $H^{2 m+2}(M)$ namely $Q_{m+1}=0, \pi^{*}(p(c))$ must be a multiple of $Q_{m+1}$. But $H^{*}\left(B G_{p}\right)=H^{*}\left(M_{G}\right)$ is freely generated by the $y_{i}$ and $Q_{j}, j>1$, and so this is possible only if $\pi^{*}(p(c))=0$. Hence $\pi^{*}(u)$ extends $Q_{m+1}$ and the argument may be completed as before. The Leray-Hirsch theorem implies that $\pi^{*}(u)$ may be written uniquely as

$$
\pi^{*}(u)=\sum_{\nu} \pi^{*}\left(u_{\nu}\right) \widetilde{b}_{\nu} \in H^{*}\left(M_{\mathcal{H}}\right),
$$

where $u_{\nu} \in H^{*}(B \mathcal{H})$. Comparing with the expression previously found for $Q_{m+1}$ we see that a multiple of $u_{\nu_{i}}$ extends a class which equals $c_{i}$ modulo products of $c_{j}, j<i$. An easy inductive argument now shows that each $c_{i}$ must extend to $B \mathcal{H}$. This completes the proof for Grassmannians.

The proof for the flag manifold $M\left(m_{1}, \ldots, m_{k}\right), k>2$, is very similar. We denote by $x_{i}$ the Chern classes of the universal bundle $E_{1} \rightarrow B U\left(m_{1}\right)$ and by $y_{\alpha i}$ the Chern classes of $E_{\alpha} \rightarrow B U\left(m_{\alpha}\right)$ for $2 \leq \alpha \leq m$. Further let $y_{i}$ be the Chern classes of the sum $F:=E_{2} \oplus \cdots \oplus E_{m}$. Since $E_{1} \oplus F$ is pulled back from $B U(\ell)$ it is trivial on $M$. Further the equation (4.3) holds as before. It should be interpreted as first defining the $x_{i}$ in terms of the generators $y_{\alpha j}$ for $H^{*}(M)$ and then giving the relations in $H^{*}(M)$. The rest of the argument goes through without essential change.

\section{The evaluation map and Whitehead products}

In this section we consider a nontrivial $S^{1}$ action $\lambda$ that is inessential in $\mathcal{G}$. For simplicity, we suppose throughout this section that $M$ is simply connected, though versions of the first lemmas extend to the general case. We shall suppose either that we are in the symplectic category so that $\mathcal{G}=\operatorname{Ham}(M, \omega)$ or that $(M, a)$ is c-symplectic, the action is smooth, and $\mathcal{G}:=\operatorname{Diff}_{0}(M)$. Let $\rho \in$ 
$\pi_{3}(\mathcal{G})$ be the nonzero element constructed in Theorem 1.1. Our first aim is to understand what it means for $\operatorname{ev}_{*}(\rho) \in \pi_{3}(M)$ to vanish.

We denote by $\partial$ the boundary map in the long exact sequence of the fibration

$$
\mathcal{G}_{p} \longrightarrow \mathcal{G} \stackrel{\text { ev }}{\longrightarrow} M .
$$

Lemma 5.1 Let $M, \mathcal{G}$ be as above and suppose that $\lambda$ is inessential in $\mathcal{G}$ but essential in $\mathcal{G}_{p}$. Then there is a unique $\alpha \in \pi_{2}(M) \otimes \mathbb{Q}$ such that $\partial \alpha=\lambda$. Moreover, $h(\alpha) \in H_{2}(M)$ is nonzero.

Proof The first statement holds because the evaluation map

$$
\mathrm{ev}_{*}: \pi_{2}(\mathcal{G}) \otimes \mathbb{Q} \rightarrow \pi_{2}(M) \otimes \mathbb{Q}
$$

is zero so that $\partial$ is injective. The second is an immediate consequence of the Hurewicz theorem: because $\pi_{1}(M)=0$ the map $\pi_{2}(M) \otimes \mathbb{Q} \rightarrow H_{2}(M)$ is an isomorphism.

Note that the element $\alpha$ may depend on the choice of $p$. For example the loop in $\operatorname{Ham}\left(\mathbb{C P}^{2}, \omega\right)$ given by $\left[z_{0}: z_{1}: z_{2}\right] \mapsto\left[e^{2 \pi i t} z_{0}: e^{-2 \pi i t} z_{1}: z_{2}\right]$ is nullhomotopic in $\mathcal{G}_{p}$ when $p=[0: 0: 1]$ but is essential when $p=[1: 0: 0]$. Therefore if we work with the corresponding diagonal circle action on $M=\mathbb{C P}^{2} \times \mathbb{C P}^{2}$ we can find points $p_{1}, p_{2}$ such that $\lambda$ is essential in $\mathcal{G}_{p_{i}}$ for $i=1,2$ but the elements $\alpha_{1}, \alpha_{2}$ are different.

Lemma 5.2 Let $\lambda, \alpha$ and $\rho:=\{\lambda, \lambda\}$ be as in Lemma 5.1 Then $\operatorname{ev}_{*}(\rho)$ is the Whitehead product $[\alpha, \alpha]$.

Proof Consider the fibration $\Omega M \rightarrow \widetilde{\mathcal{G}}_{p} \rightarrow \mathcal{G}$, where $\Omega M$ is the based loop space of $M$ and $\widetilde{\mathcal{G}}_{p}$ is the space of all pairs $(h, \gamma)$ where $h \in \mathcal{G}$ and $\gamma$ is a path in $M$ from the base point $p$ to $h(p)$. The choice of a contraction $\tilde{\lambda}$ of $\lambda$ in $\mathcal{G}$ determines a homotopy from $\lambda$ to a loop $\ell: s \mapsto \ell(s)$ in $\Omega M$ : if $\widetilde{\lambda}$ is given by a map

$$
\begin{aligned}
& {[0,1] \times[0,1] \rightarrow \mathcal{G}, \quad(s, \nu) \mapsto \widetilde{\lambda}(s, \nu),} \\
& \widetilde{\lambda}(s, 0)=\widetilde{\lambda}(0, \nu)=\widetilde{\lambda}(1, \nu)=i d, \quad \widetilde{\lambda}(s, 1)=\lambda(s),
\end{aligned}
$$

then for each $s \in[0,1], \ell(s)$ is the loop $\nu \mapsto \widetilde{\lambda}(s, \nu)(p)$.

It suffices to show that the image of $\rho$ under the boundary map $\partial: \pi_{3}(\mathcal{G}) \rightarrow$ $\pi_{2}(\Omega M)$ is the Samelson product $\langle\ell, \ell\rangle$ since this is the desuspension of $[\alpha, \alpha]$. 
But it follows from the definition of $\rho$ as a map $D^{2} \times S^{1} / \sim \longrightarrow \mathcal{G}$ that $\partial \rho$ is represented by the map

$$
S^{1} \times S^{1} / \sim \longrightarrow \Omega M, \quad(s, t) \mapsto\langle\widetilde{\lambda}(s, \cdot), \lambda(t)\rangle(p),
$$

where $(s, t) \in S^{1} \equiv \mathbb{R} / \mathbb{Z}$. But this is homotopic to the map $\langle\ell, \ell\rangle:(s, t) \mapsto$ $\gamma(s, t)$ where

$$
\gamma(s, t)(\nu):=\langle\widetilde{\lambda}(s, \nu), \widetilde{\lambda}(t, \nu)\rangle(p)
$$

via the homotopy $\gamma_{r}(s, t)(\nu):=\langle\widetilde{\lambda}(s, \nu), \tilde{\lambda}(t, r+\nu(1-r))\rangle(p)$.

A similar argument shows that if the product $\left\{f, f^{\prime}\right\}$ is as defined in Proposition 2.1 then $\operatorname{ev}_{*}\left(\left\{f, f^{\prime}\right\}\right)=\left[\alpha, \alpha^{\prime}\right]$, provided that $f, f^{\prime}$ both vanish in $\mathcal{G}$ so that they correspond to elements $\alpha, \alpha^{\prime} \in \pi_{*}(M)$. If only $f$ vanishes in $\mathcal{G}$, $\mathrm{ev}_{*}\left(\left\{f, f^{\prime}\right\}\right)$ need not be a Whitehead product. For example, in the case of the action of $S U(n+1)$ on $\mathbb{C P}^{n}$ we have (in the notation of Lemma 2.5) $\operatorname{ev}_{*}\left(\left\{\lambda_{1}, \lambda_{3}\right\}\right)=0$, but $\operatorname{ev}_{*}\left(\left\{\lambda_{1}, \lambda_{2 n-1}\right\}\right) \neq 0$. In this particular example, $\operatorname{ev}_{*}\left(\left\{\lambda_{1}, \lambda_{2 n-1}\right\}\right)$ is a higher order Whitehead product. We now investigate the extent to which this generalizes. To get clean statements we need to assume that the homotopy of $M$ has some of the characteristics of $\mathbb{C P}^{n}$. For the first result we only need $M$ to be simply connected, but later on need more assumptions.

Proposition 5.3 Let $\lambda$ be a nontrivial $S^{1}$-action on a simply connected csymplectic manifold $(M, a)$. Suppose that there is $\alpha \in \pi_{2}(M)$ such that $\partial \alpha=$ $\lambda \in \pi_{1}\left(\mathcal{G}_{p}\right)$. Then $\operatorname{ev}_{*}(\rho) \neq 0$ if and only if any element $c \in H^{2}(M)$ such that $c(\alpha) \neq 0$ has the property that $c^{2}=0$ modulo the ideal $\mathcal{I}$ in $H^{*}(M)$ generated by the kernel of $\alpha^{*}: H^{2}(M) \rightarrow H^{2}\left(S^{2}\right)$.

Proof If $\operatorname{ev}_{*}(\rho)=0$ then Lemma 5.2 implies that the map $\alpha \vee \alpha: S^{2} \vee S^{2} \rightarrow M$ extends to $S^{2} \times S^{2}$. Hence if $c \in H^{2}(M)$ is such that $c(\alpha) \neq 0$ its square $c^{2}$ has nonzero pullback to $S^{2} \times S^{2}$ and hence is nonzero modulo $\mathcal{I}$.

The converse follows from from Lemma 5.2 and minimal model theory. When building a minimal model $\mathcal{A}:=\left(A^{k}, d\right)_{k \geq 0}$ for $H^{*}(M)$ we may choose a basis $c_{0}, \ldots c_{k}$ for the 2-cochains $A^{2}$ such that $c_{i}(\alpha)=0, i>0$. The differential $d: A^{3} \rightarrow\left(A^{2}\right)^{2} \subset A^{4}$ is dual to the Whitehead product $\pi_{2}(M) \times \pi_{2}(M) \rightarrow$ $\pi_{3}(M)$. We may choose a basis for $\pi_{3}(M)$ whose first element is $\operatorname{ev}_{*}(\rho)$. Then if $r \in A^{3}$ is the first element in the dual basis, $d r=\sum \mu_{i j} c_{i} c_{j}$ where $\mu_{00}=1$. Hence $d r=\left(c_{0}\right)^{2}$ modulo $\mathcal{I}$. 
We now explore what happens when $e v_{*}(\rho)=0$. This means that the Whitehead product $[\alpha, \alpha]$ is zero. Thus we can consider the higher order Whitehead product $[\alpha, \alpha, \alpha] \subset \pi_{5}(M)$ which is defined as follows (see [3] for details). Let $W \subset S^{2} \times S^{2} \times S^{2}$ denote the fat wedge, that is it consists of triples with at least one coordinate at the base point. Then $[\alpha, \alpha, \alpha]:=\left\{f_{*}(u)\right\} \subset \pi_{5}(M)$, where $f: W \rightarrow M$ ranges over the set of all extensions of $\alpha \vee \alpha \vee \alpha$ and $u \in \pi_{5}(W)$ is a generator. Since $[\alpha, \alpha]=0$ this set is nonempty. Moreover, because $W$ consists of the wedge of three copies of $\left(S^{2} \times S^{2}\right) \vee S^{2}, f$ is determined by the way in which $\alpha \vee \alpha$ is extended to $S^{2} \times S^{2}$, which can vary by an element $\beta \in \pi_{4}(M)$. Therefore $[\alpha, \alpha, \alpha]$ is a coset of the subgroup

$$
H:=\left\{[\alpha, \beta]: \beta \in \pi_{4}(M)\right\} \subset \pi_{5}(M) .
$$

We say that $[\alpha, \alpha, \alpha]$ is nonzero if this coset does not contain the zero element. Hence $[\alpha, \alpha, \alpha]=0$ if and only if some extension $f: W \rightarrow M$ of $\alpha \vee \alpha \vee \alpha$ extends further to $S^{2} \times S^{2} \times S^{2}$.

In order to interpret the vanishing of $\mathrm{ev}_{*}(\rho)=0$ in cohomological terms (rather than in terms of the minimal model) we need to make some further simplifying assumptions about the homotopy type of $M$. We assume below that $\pi_{3}(M)=$ 0 , so that $[\alpha, \alpha]=0$ a fortiori. Note that this hypothesis is satisfied by all generalized flag manifolds $M\left(m_{1}, \ldots, m_{k}\right)$ with $m_{1}>1$.

Proposition 5.4 Let $\pi_{1}(M)=\pi_{3}(M)=0$ and $\lambda: S^{1} \rightarrow \mathcal{G}_{p}$ be a nontrivial action as in Proposition [5.3. Suppose that there is $\alpha \in \pi_{2}(M)$ such that $\partial \alpha=\lambda \in \pi_{1}\left(\mathcal{G}_{p}\right)$. Consider $c \in H^{2}(M)$ such that $c(\alpha) \neq 0$. Then the higher Whitehead product $[\alpha, \alpha, \alpha]$ is nonzero if and only if $c^{3} \in \mathcal{I}$, where $\mathcal{I}$ is as in Proposition 5.3.

Proof One direction is trivial. Namely, if $[\alpha, \alpha, \alpha]=0$ then $\alpha \vee \alpha \vee \alpha$ extends to $S^{2} \times S^{2} \times S^{2}$ and so $c^{3} \notin \mathcal{I}$.

Now assume that $[\alpha, \alpha, \alpha] \neq 0$. Chose some extension of $\alpha \vee \alpha$ to $S^{2} \times S^{2}$ and using this define $f: W \rightarrow M$ in a symmetric way. Denote $\gamma_{0}:=f_{*}(u)$. By hypothesis the span in $\pi_{5}(M)$ of the Whitehead products $\left[\alpha, \pi_{4}(M)\right]$ does not include $\gamma_{0}$.

The minimal models of the spaces under consideration have the following forms:

- $\mathcal{A}(W)=\Lambda\left(x_{1}, x_{2}, x_{3} ; y_{1}, y_{2}, y_{3} ; w ; \ldots\right)$ where $\operatorname{deg} x_{i}=2, \operatorname{deg} y_{i}=3, \operatorname{deg} w=5$ and the nontrivial differentials are $d y_{i}=x_{i}^{2}, d w=x_{1} x_{2} x_{3}$ and enough others in higher degrees to cancel out the cohomology. Note that this minimal model is infinitely generated. 
- $\mathcal{A}(M)=\Lambda\left(c_{0}, c_{1}, \ldots, c_{k} ; v_{1}, \ldots, v_{\ell} ; z \ldots\right)$,

where $\operatorname{deg} c_{i}=2, \operatorname{deg} v_{i}=4, \operatorname{deg} z=5$ and the first nontrivial differential is given by $d z=\sum a_{i j k} c_{i} c_{j} c_{k}+\sum a_{\ell m} c_{\ell} v_{m}$, for some $a_{i j k}, a_{\ell m} \in \mathbb{Q}$. Moreover, $c_{0}(\alpha)=1$ and $c_{i}(\alpha)=0$ for $i>0$.

According to the above discussion about $\left[\alpha, \pi_{4}(M)\right]$ we can choose $z \in A^{5}(M)$ so that $z$ vanishes on all the elements $[\alpha, \beta]$ for $\beta \in \pi_{4}(M)$ but $z\left(\gamma_{0}\right)=1$. ¿From this we draw two conclusions. Firstly, because the quadratic part of $d z$ is dual to the Whitehead product, $d z$ includes no terms of the form $c_{0} v_{m}$. In other words $a_{0 m}=0$ for all $m$ and $d z=\sum a_{i j k} c_{i} c_{j} c_{k}$. Secondly, because $f^{*}(z)=w+\sum b_{i j} x_{i} y_{j}$ and $f^{*}\left(c_{i}\right)=0, i>0$,

$$
\begin{aligned}
0 & \neq x_{1} x_{2} x_{3}+\sum b_{i j} x_{i} x_{j}^{2} \\
& =d\left(f^{*}(z)\right)=f^{*}(d z)=a_{000} f^{*}\left(c_{0}^{3}\right) .
\end{aligned}
$$

Therefore $a_{000} \neq 0$, and the relation in cohomology given by setting $d z$ to zero says that $\left[c_{0}\right]^{3} \in \mathcal{I}$.

Our final result concerns the question of whether $[\alpha, \alpha, \alpha] \cap \operatorname{Im} \operatorname{ev}_{*} \neq \emptyset$, that is whether the Whitehead product contains elements from the image of the evaluation map. Again, we need to strengthen the hypothesis that $[\alpha, \alpha]=0$. The latter implies that $\rho$ lifts to an element $\rho_{1} \in \pi_{3}\left(\mathcal{G}_{p}\right)$, and we now assume that this lift can be chosen so that the Samelson product $\left\langle\lambda, \rho_{1}\right\rangle$ vanishes in $\mathcal{G}_{p}$. By Lemma 2.5 this will hold if, for example, $\lambda$ is an essential circle in $U(2) \subset$ $G_{p}$ and the map $U(2) \rightarrow G$ factors through $S U(2)$. Again, this hypothesis holds for flag manifolds $M\left(m_{1}, \ldots, m_{k}\right)$ with $m_{1}>1$, and in particular for $\mathbb{C P}^{n}$.

Proposition 5.5 Let $\lambda$ be a nontrivial $S^{1}$-action on a simply connected $c$ symplectic manifold $(M, a)$, and let $\mathcal{G}:=\operatorname{Ham}(M, a)$. Suppose that there is $\alpha \in \pi_{2}(M)$ such that $\partial \alpha=\lambda \in \pi_{1}\left(\mathcal{G}_{p}\right)$ and $[\alpha, \alpha]=0$. Suppose further that $\rho$ has a lift $\rho_{1}$ to $\mathcal{G}_{p}$ such that $\left\langle\lambda, \rho_{1}\right\rangle=0$ in $\mathcal{G}_{p}$. Then if $[\alpha, \alpha, \alpha] \neq 0$ in $\pi_{5}(M) \otimes \mathbb{Q}$ there is a nonzero element in the intersection of $[\alpha, \alpha, \alpha]$ with the image of $\mathrm{ev}_{*}: \pi_{5}(\mathcal{G}) \rightarrow \pi_{5}(M)$.

Proof Consider the commutative diagram:

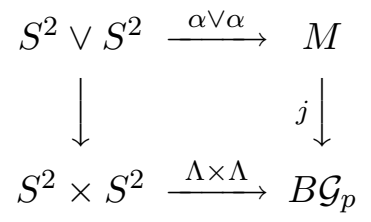


By assumption, the map $\alpha \vee \alpha$ has some extension $\phi$ to $S^{2} \times S^{2}$. However, because $\pi \circ(\Lambda \times \Lambda)$ is nontrivial (where $\pi: B \mathcal{G}_{p} \rightarrow B \mathcal{G}$ ) we cannot choose $\phi$ to make this diagram commute. On the other hand, because $\pi \circ(\Lambda \times \Lambda)$ is the composite of the quotient map $S^{2} \times S^{2} \rightarrow S^{4}$ with a representative of $\bar{\rho}$, we can adjust $\Lambda \times \Lambda$ on the top cell of $S^{2} \times S^{2}$ by a lift $\tau$ of $-\bar{\rho}$ to a map $f:=\Lambda \times \Lambda \# \tau: S^{2} \times S^{2} \rightarrow B \mathcal{G}_{p}$ that does lift to $M$. Thus we can arrange that $f=j \circ \phi$ for suitable $\phi$, ie, that the following diagram commutes:

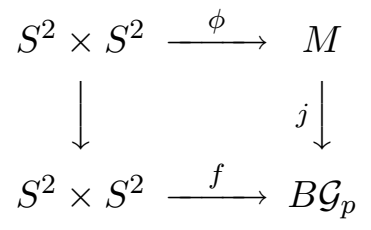

Our assumptions imply that we may choose $\tau$ so that in addition $[\Lambda, \tau]=0$ in $B \mathcal{G}_{p}$. Consider the commutative diagram:

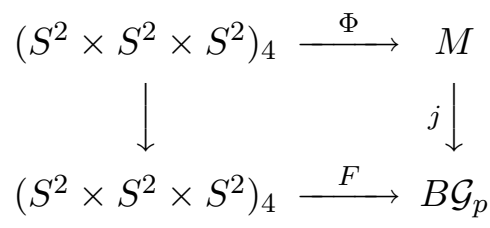

where $\Phi$ is given by $\phi$ and $F$ is given by $f$. Since $\Lambda \vee \Lambda \vee \Lambda$ extends to the product map $\Lambda \times \Lambda \times \Lambda$, the obstruction to extending $F$ to the product $S^{2} \times S^{2} \times S^{2}$ is $3[\Lambda, \tau]$ and so vanishes. Therefore the obstruction to extending $\Phi$ is an element in $[\alpha, \alpha, \alpha]$ that has zero image in $\pi_{*}\left(B \mathcal{G}_{p}\right)$ and hence lies in the image of $\mathrm{ev}_{*}$.

If $[\alpha, \alpha, \alpha]=0$ one could explore yet higher order products. However, to make sense of the results one would need further assumptions on $M$ and the behavior of $\lambda$.

\section{References}

[1] M Abreu, D McDuff, Topology of symplectomorphism groups of rational ruled surfaces, J. Amer. Math. Soc. 13 (2000) 971-1009 MathReview

[2] C Allday, Examples of circle actions on symplectic spaces, from: "Homotopy and Geometry (Warsaw 1997)", Banach Center Publications 45, Institute of Mathematics, Polish Acad. Sci. Warszawa (1998) MathReview

[3] $\mathbf{P}$ Andrews, M Arkowitz, Sullivan's minimal models and higher order Whitehead products, Canad. J. Math. 30 (1978) 961-982 MathReview 
[4] S Anjos, Homotopy type of symplectomorphism groups of $S^{2} \times S^{2}$, Geom. Topol. 6 (2002) 195-218 MathReview

[5] S Anjos, G Granja, Homotopy decomposition of a group of symplectomorphisms of $S^{2} \times S^{2}$, Topology 43 (2004) 599-618 MathReview

[6] A Blanchard, Sur les variétés analytiques complexes, Ann. Sci. Ec. Norm. Sup. 73 (1956) 157-202 MathReview

[7] S Gal, J Kȩdra, Symplectic configurations, in preparation

[8] V Ginzburg, V Guillemin, Y Karshon, Moment maps, cobordisms and Hamiltonian group actions, Mathematical Surveys and Monographs 98, AMS (2002) MathReview

[9] D Gottlieb, Evaluation subgroups of homotopy groups, Amer. J. Math. 91 (1969) 729-756 MathReview

[10] M Gromov, Pseudoholomorphic curves in symplectic manifolds, Invent. Math. 82 (1985) 307-347 MathReview

[11] V Guillemin, E Lerman, S Sternberg, Symplectic fibrations and multiplicity diagrams, Cambridge University Press (1999) MathReview

[12] V Guillemin, S Sternberg, Supersymmetry and Equivariant de Rham Theory, Springer (1999) MathReview

[13] T Januszkiewicz, J Kẹdra, Characteristic classes of smooth fibrations, arXiv:math.SG/0209288

[14] F Lalonde, D McDuff, Symplectic structures on fiber bundles, Topology 42 (2003) 309-347 MathReview

[15] D McDuff, Symplectic diffeomorphisms and the flux homomorphism, Invent. Math. 77 (1984) 353-66 MathReview

[16] D McDuff, A survey of topological properties of groups of symplectomorphisms, from: "Topology, Geometry and Quantum Field Theory, Proceedings of 2002 Symposium in honor of G B Segal", (U L Tillmann, editor), LMS Lecture Notes series 308, Cambridge Univ. Press (2004) 173-193 MathReview

[17] D McDuff, Enlarging the Hamiltonian group, preprint (2004)

[18] D McDuff, D Salamon, Introduction to Symplectic Topology, 2nd edition, Oxford Univ. Press, Oxford, (1998) MathReview

[19] D McDuff, S Tolman, Topological properties of Hamiltonian circle actions, arXiv:math.SG/0404338

[20] D McDuff, S Tolman, in preparation

[21] K Ono, Floer-Novikov cohomology and the flux conjecture, preprint (2004)

[22] A G Reznikov, Characteristic classes in symplectic topology, Selecta Math 3 (1997) 601-642 MathReview

[23] P Seidel, Lectures on four dimensional Dehn twists, arXiv:math.SG/0309012

[24] A Tralle, J Oprea, Symplectic manifolds with no Kähler structure, Springer Lecture Notes series 1661 (1997) MathReview 